\title{
Quasi Hopf quantum symmetry in quantum theory
}

\author{
Gerhard Mack * and Volker Schomerus \\ II. Institut für Theoretische Physik, Unicersität Hamburg, D-2000 Hamburg, Germany
}

Received 6 May 1991

(Revised 17 July 1991)

Accepted for publication 12 September 1991

\begin{abstract}
In quantum theory, internal symmetries more general than groups are possible. We show that quasi-triangular quasi Hopf algebras $\mathscr{G}^{*}$ ("quasi quantum groups") as introduced by Drinfeld [1] permit a consistent formulation of a transformation law of states in the physical Hilbert space $\not{F}$. of invariance of the ground state, and of a transformation law of field operators which is consistent with local braid relations of field operators which generalise those proposed by Fröhlich [2]. All this remains true when Drinfeld's axioms are suitably weakened in order to build in truncated tensor products. Conversely, all the axioms of a weak quasi-triangular quasi Hopf algebra are motivated from what physics demands of a symmetry. Unitarity requires in addition that $\mathscr{G}^{*}$ admits a $*$-operation with certain properties. Invariance properties of Green functions follow from invariance of the ground state and covariance of field operators as usual. Covariant adjoints and covariant products of field operators can be defined. The $R$-matrix elements in the local braid relations are in general operators in $\mathscr{H}$. They are determined by the symmetry up to a phase factor. Quantum group algebras like $\mathrm{U}_{q}\left(\mathrm{sl}_{2}\right)$ with $|q|=1$ are examples of symmetries with special properties. We show that a weak quasi-triangular quasi Hopf algebra $G^{*}$ is canonically associated with $\mathrm{U}_{q f}\left(\mathrm{sl}_{2}\right)$ if $q^{p}=1$. We argue that these weak quasi Hopf algebras are the true symmetries of minimal conformal models. Their dual algebras $\mathscr{G}$ ' ("functions on the group") are neither commutative nor associative.
\end{abstract}

\section{Introduction}

In quantum physics, states $|\psi\rangle$ are elements of a Hilbert space $\mathscr{H}$, and (i) Elements of a symmetry act as transformations of physical states (i.e. as operators in $\left.\mathscr{H}^{\prime}\right)|\psi\rangle \mapsto\left|\psi^{\prime}\right\rangle$;

(ii) The transformation law is consistent with statistics;

(iii) There is a relation between spin and statistics. Bosons have spin $s=0,1, \ldots$, fermions have spin $s=\frac{1}{2}, \frac{3}{2}, \ldots$, but other values for $s$ are possible in 2 space dimensions, and are associated with braid group statistics.

In this paper we assume that braid group statistics is implemented through local braid relations among the field operators which create and annihilate particles or

* E-mail: I02MAC(a DHHDESY3.BITNET 
excitations [2]. We examine what symmetries are consistent with this assumption. They are called quantum symmetries and are not groups in general.

To find signs of (nonabelian) quantum symmetry in nature, experimental solid state physicists should look for degenerate multiplets of excitations with non-halfinteger fractional spin in two-dimensional quantum systems.

It appears that the possibility of quantum symmetries is special to quantum theory. In classical mechanics, symmetries are groups of transformations which act on phase space. The observables form a commutative algebra $\mathscr{A}$ which consists of functions $f$ on phase space; the hamiltonian is one of these. In quantum mechanics, the points of phase space have gone away, but a (noncommutative) algebra of observables or field operators persists. It is surprising that it took 60 years to discover that quantum theory admits a larger class of symmetries than groups. Groups are topological spaces $G$ whose points are the group elements $a$. When the points of phase space on which the group elements act have gone away, one does not need the group elements $a$ any more either. It is said that "quantum theory may be regarded as mechanics on noncommutative phase space" [9] and that "quantum groups are the symmetries of noncommutative spaces" $[10,11]$. But actually they are only examples with special properties as we shall see.

The reader is invited to think of (second quantized) nonrelativistic many-particle systems or of relativistic quantum field theories as examples of quantum mechanical systems.

When there is a symmetry group $G$, the Hilbert space of physical states carries a (unitary) representation of an algebra $\mathscr{G}^{*}$ (the group algebra) which contains the group elements as distinguished elements that generate it. $\mathscr{G}^{*}$ is the dual of the commutative algebra $\mathscr{G}$ of functions on the group G, i.e. its elements $\phi \in \mathscr{G}^{*}$ are linear maps

$$
\phi: \quad \mathscr{G} \mapsto \mathbb{C}
$$

Actually $\mathscr{G}$ is a "bi-algebra" * and this yields a product in $\mathscr{G}^{*}$ known as the convolution product. The notion of invariance of the ground state or vacuum can be formulated in this language, without reference to group elements, and also the transformation law of field operators which create particles or excitations. This is all that is needed to formulate the notion of a symmetry and to extract from it the usual consequences (sect. 2).

It is therefore possible to admit as symmetry algebra $\mathscr{G}^{*}$ the dual of a more general bi-algebra $\mathscr{G}$ than the commutative algebra of functions on a group (sect. 3). Quantum groups [7] are among these more general symmetries. They are associated with a special type of braid relations which involve solutions of the Yang-Baxter equations [2] (sect. 4). But this is not the most general possibility as will be seen. Nonassociative algebras $\mathscr{G}$ are also admissible when they are the dual

* A bi-algebra is an algebra with a product and a co-product, a unit element and a co-unit - see later. 
of a quasi-triangular quasi Hopf algebra $\mathscr{G}^{*}$ as introduced by Drinfeld [1]. We will exhibit the appropriate form of the local braid relations of field operators which is quasi Hopf invariant. (It involves solutions of quasi-Yang-Baxter equations.) (sect. 5). But this is still not the most general possibility. We show that all the physical requirements, including in particular validity of local braid relations of field operators, remain satisfied when Drinfeld's axioms are weakened in such a way that the truncated tensor products which have appeared in conformal field theory are accommodated (sect. 6). In this case we speak of a weak quasi-triangular quasi Hopf algebra. Conversely, all the axioms of a weak quasi-triangular quasi Hopf algebra will be motivated by properties which one wants quantum fields to have, and we believe therefore that this is the general algebraic structure of a symmetry in low dimensions.

In all cases a *-operation with certain properties is needed in order to formulate unitarity. This is a somewhat subtle issue. We will explain in some detail why quantum group algebras $\mathrm{U}_{q}\left(\mathrm{sl}_{2}\right)$ with $|q|=1$ are consistent with this requirement, and how adjoints of field opertors can be defined which transform covariantly (sect. 7).

In this paper, $\mathscr{G}^{*}$ will be regarded as the fundamental symmetry algebra. Emphasis is on its representations in the Hilbert space $\mathscr{H}$ of physical states, as opposed to symmetries of Green functions. The transformation law of field operators is the same as proposed by Buchholz et al. for quantum group algebras [4]. There is an alternative picture, proposed by Reshetikhin and Smirnov [15], where $\mathscr{H}$ is regarded as a co-module for $\mathscr{G}$. The relation between both interpretations will be explained in sect. 8 .

Models with quantum groups as symmetries were studied by many authors, [4,5,13-17,19-22] and others. Integrable models, conformal field theory, and the massive sine-Gordon model provide examples. Green functions of an orbifold model with a quasi Hopf algebra as symmetry were examined by Dijkgraaf et al. [3].

Some pedagogical material that is well known to experts is included in this paper for the benefit of the reader. But the construction of quasi Hopf covariant products (5.4) and adjoints (7.2) of field operators, and the resulting quasi Hopf invariant form of local braid relations of field operators (5.7)ff. are believed to be new, and so is the weaker form of the quasi Hopf axioms which permits to incorporate truncated tensor products, and the identification of what we believe to be the general algebraic structure of a symmetry in low dimensions.

Sect. 9 is a review of known facts about the quantum group algebras $\mathrm{U}_{q}\left(\mathrm{sl}_{2}\right)$.

Our results on the incorporation of truncated tensor products are in sect. 6 and 10. We show in sect. 10 that a weak quasi-triangular quasi Hopf algebra $\mathscr{G}^{*}$ is associated in a canonical way to every quantum group algebra $\mathrm{U}_{q}\left(\mathrm{sl}_{2}\right)$ with $q^{p}=1$. The physical representations of $\mathrm{U}_{q}\left(\mathrm{sl}_{2}\right)$ are representations of $\mathscr{G}^{*}$, and the co-product of $\mathscr{G}^{*}$ yields the truncated tensor product of physical representations 
of $\mathrm{U}_{q}\left(\mathrm{sl}_{2}\right)$. Since this truncated tensor product is the "physical" tensor product in conformal models [19], it appears appropriate to regard $\mathscr{G}^{*}$ rather than $\mathrm{U}_{q}\left(\mathrm{sl}_{2}\right)$ as the true symmetry of the conformal models. Our results on local braid relations imply for instance that an appropriate form of local braid relations for field operators holds in the whole physical Hilbert space of the conformal Ising model [5] *. There is no need to introduce unphysical states to achieve this. This is in contrast with what happens when one interprets the full algebra $\mathrm{U}_{q}\left(\mathrm{sl}_{2}\right)$ as a symmetry, instead of the appropriate algebra $\mathscr{G}^{*}=\mathrm{U}_{q}\left(\mathrm{sl}_{2}\right) / \mathscr{F}$ with a non-co-associative coproduct as proposed here. The $\mathrm{U}_{q}\left(\mathrm{sl}_{2}\right)$ braid relations are either only

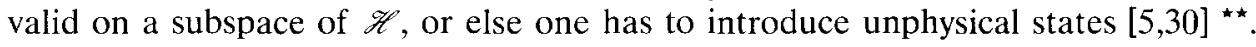

The weak quasi Hopf character of the symmetry affects not only the braid relations, but also the operator product expansions. It is the (nonassociative) covariant product of field operators which involves numerical Clebsch-Gordan coefficients. Upon transition to (associative) ordinary products, these numerical Clebsch-Gordan coefficients get multiplied with representation operators of the symmetry algebra in the physical Hilbert space.

In a weak quasi-triangular quasi Hopf algebra there exists an element $\varphi \in \mathscr{G}^{*} \otimes$ $\mathscr{G}^{*} \otimes \mathscr{G}^{*}$ which assures quasi-associativity of tensor products. Its construction uses solutions of the pentagon and hexagon identities. These identities appeared first in conformal field theory [34].

It is instructive to note that supersymmetry can be regarded as a special case of a quantum symmetry (sect. 11).

By definition, a nontrivial transformation law under a nonabelian quantum symmetry is consistent with local braid relations, but not in general with local Bose commutation relations. Observables are therefore invariant, i.e. quantum symmetries are gauge symmetries (of first kind). The hamiltonian is the integral of a local Bose field. The important question arises therefore, whether nonabelian quantum symmetry can be broken at all by a perturbation (or spontaneously), and under what conditions. We are not prepared to discuss this question.

\section{Abstraction from symmetry groups}

We begin with well-known facts about group representations in quantum mechanics. The idea will be to reformulate them so that the group elements can be discarded in the end.

First we remind the reader that the Hilbert space $\mathscr{H}$ carries a unitary representation of the group algebra $\mathscr{G}^{*}$. Then we will deduce the transformation law of field operators under $\mathscr{G}^{*}$. This will involve the co-multiplication. The statement of

* The fields which satisfy these braid relations are precisely those constructed in ref. [5]. The proof is spelled out in ref. [6].

** The unphysical states may transform nontrivially under the ideal $\mathscr{g}$ which we propose to factor out. 
invariance of the ground state will involve the co-unit. The transformation law of adjoints of field operators will involve the antipode. And the transformation law of states can be recovered from the transformation law of field operators and invariance of the ground state.

To state all this in more detail, we begin with the definition and representations of $\mathscr{G}^{*}$. Let $\mathrm{G}$ be a compact symmetry group of a quantum mechanical system. Then there will be a unitary operator $U(a)$ associated with every group element $a$. These operators act in the Hilbert space of physical states,

$$
U(a): \quad \mathscr{H} \mapsto \mathscr{H} .
$$

Let $\mathscr{G}$ be the commutative algebra of ("good") functions $f$ on $\mathrm{G}$,

$$
f: \quad \mathrm{G} \mapsto \mathbb{C} .
$$

The product $\cdot$ in $\mathscr{G}$ is defined by pointwise multiplication, $f \cdot g(a)=f(a) g(a)$.

The dual $\mathscr{G}^{*}$ of $\mathscr{G}$ consists of linear maps

$$
\phi: \quad \mathscr{G} \mapsto \mathbb{C} .
$$

The group elements $a \in \mathrm{G}$ can be regarded as special elements of $\mathscr{G}^{*}$ by virtue of the assignment

$$
a(f)=f(a) \text { for } f \in \mathscr{G} .
$$

Compact symmetry groups admit a volume element $\mathrm{d} a$ known as Haar measure, which is uniquely determined by the requirement of invariance and normalization. Elements $\phi$ of $\mathscr{G}^{*}$ are determined by generalized functions [36] $\Phi$ on G. We use the shorthand notation

$$
\phi=\int_{\mathrm{G}} \mathrm{d} a \Phi(a) a
$$

It follows that

$$
\phi(f)=\int \mathrm{d} a \Phi(a) a(f)=\int \mathrm{d} a \Phi(a) f(a)
$$

by eq. (2.3). A group element $a$, regarded as an element of $\mathscr{G}^{*}$, is identified with the $\delta$-function of $\mathrm{G}$ supported at $a$.

The product in $\mathscr{G}^{*}$ is given by convolution of generalized functions, since

$$
\phi_{1} \phi_{2}=\int \mathrm{d} a \int \mathrm{d} b \Phi_{1}(a) \Phi_{2}(b) a b=\int \mathrm{d} c\left(\Phi_{1} * \Phi_{2}\right)(c) c
$$

with

$$
\left(\Phi_{1} * \Phi_{2}\right)(c)=\int \mathrm{d} a \Phi_{1}(a) \Phi_{2}\left(a^{-1} c\right)
$$


For group elements, regarded as elements of $\mathscr{G}^{*}$ in the manner just explained, this definition agrees with the product definition in $\mathrm{G}$.

The algebra $\mathscr{G}$ admits a unit element 1 , the constant function 1 on $\mathrm{G}$. One defines a co-unit on $\mathscr{G}^{*}, \epsilon: \mathscr{G}^{*} \mapsto \mathbb{C}$, which is a homomorphism of algebras and is defined by

$$
\epsilon(\phi)=\phi(1)=\int \mathrm{d} a \Phi(a)
$$

Later on we shall need also the co-product $\Delta$ on $\mathscr{G}^{*}$. It is a linear map $\Delta$ : $\mathscr{G}^{*} \mapsto \mathscr{G}^{*} \otimes \mathscr{G}^{*}$ and is explicitly defined by reference to the product in $\mathscr{G}$, viz.

$$
\Delta(\phi)(f, g)=\phi(f \cdot g) .
$$

When $\mathscr{F}$ is a space of functions of one variable, then $\mathscr{F} \otimes \mathscr{F}$ consists of functions of two variables. Therefore elements of $\mathscr{G}^{*} \otimes \mathscr{G}^{*}$ may be regarded as maps of pairs of functions $(f, g)$ into complex numbers. $\mathscr{G}^{*} \otimes \mathscr{G}^{*}$ is an algebra with product $\left(\xi_{1} \otimes \xi_{2}\right)\left(\eta_{1} \otimes \eta_{2}\right)=\xi_{1} \eta_{1} \otimes \xi_{2} \eta_{2}$, and $\Delta$ is in fact a homomorphism of algebras.

The representation of $\mathrm{G}$ induces a representation of the algebra $\mathscr{G}^{*}$ by operators

$$
U(\phi): \quad \mathscr{H} \mapsto \mathscr{H}
$$

in the Hilbert space of physical states, viz.

$$
U(\phi)=\int_{\mathrm{G}} \mathrm{d} a \Phi(a) U(a)
$$

The representation property holds,

$$
U\left(\phi_{1} \phi_{2}\right)=U\left(\phi_{1}\right) U\left(\phi_{2}\right)
$$

Unitarity of the representation translates into

$$
U\left(\phi^{*}\right)=U(\phi)^{*}
$$

The action of the *-operations on elements $\phi \in \mathscr{G}^{*}$ is defined by $a^{*}=a^{-1}$, and more generally by $\phi^{*}=\int \mathrm{d} a \overline{\Phi(a)} a^{-1}$. Finally, G-invariance of the ground state $|0\rangle$ translates into

$$
U(\phi)|0\rangle=|0\rangle \epsilon(\phi)
$$

We ended up with statements which involve only the algebra $\mathscr{G}^{*}$ and the *-operation and co-unit on it, but make no reference to group elements. Summing up, the Hilbert space carries a unitary representation of the algebra $\mathscr{G}^{*}$. Unitarity 
is expressed through eq. (2.11) and invariance of the ground state through eq. (2.12).

Let us now suppose that the Hilbert space of physical states is created from the ground state $|0\rangle$ by application of field operators $\Psi_{i}^{I}(r, t)$ which create particles or excitations. $I, i$ are some quantum numbers which distinguish these. Later on, $I$ will specify the transformation law (isospin, for instance) while $i$ distinguishes members of a multiplet. Let us suppose that these field operators transform covariantly under the symmetry group $\mathrm{G}$. This means that there exist finite-dimensional representations of $\mathrm{G}$ by matrices $\tau^{\prime}(a)$ such that

$$
U(a) \Psi_{i}^{I}=\Psi_{k}^{I} \tau_{k i}^{I}(a) U(a)
$$

Summation over repeated indices will be understood throughout. We will neglect to write arguments $(\boldsymbol{r}, t)$ of field operators when their values do not matter. Eq. (2.13) says that $\Psi^{I}$ is a tensor operator.

We will generalize this formula to arbitrary elements $\phi \in \mathscr{G}^{*}$. The resulting formula will involve the co-product $\Delta$. By definition $\Delta(\phi)$ is an element of $\mathscr{G}^{*} \otimes \mathscr{G}^{*}$ and is therefore of the following form. To every $\phi$ there exist sequences of elements $\phi_{p}^{1}$ and $\phi_{p}^{2}$ of elements of $\mathscr{G}^{*}$ such that

$$
\Delta(\phi)=\sum_{p} \phi_{p}^{1} \otimes \phi_{p}^{2}
$$

If $\phi$ is a group element, then there exists only one term in the sum. We may admit integrals in place of sums $\Sigma_{p}$ and use our shorthand notation to write an explicit general formula,

$$
\Delta(a)=a \otimes a \quad \text { for } a \in \mathrm{G}, \quad \Delta(\phi)=\int \mathrm{d} a \Phi(a) a \otimes a
$$

The covariance property generalizes to

$$
U(\phi) \Psi_{i}^{I}=\sum_{p} \Psi_{m}^{I} \tau_{m i}^{I}\left(\phi_{p}^{1}\right) U\left(\phi_{p}^{2}\right)
$$

$U(\phi)$ was defined in eq. (2.9) and $\tau(\phi)$ is defined in the same way.

Let us check eq. (2.16) with the use of the explicit formula (2.15). The right-hand side of eq. (2.16) will read

$$
\int \mathrm{d} a \Phi(a) \Psi_{m}^{I} \tau_{m i}^{I}(a) U(a)
$$

By virtue of eq. (2.13) this is indeed equal to

$$
U(\phi) \Psi_{i}^{I}=\int \mathrm{d} a \Phi(a) U(a) \Psi_{i}^{I}
$$


Let us now determine the transformation law of the special states

$$
|I, i\rangle=\Psi_{i}^{I}|0\rangle
$$

that follow from this. Denoting the identity map by id, co-product and co-unit have the property that

$$
(\mathrm{id} \otimes \epsilon) \Delta=\mathrm{id} .
$$

This means that validity of eq. (2.14) implies

$$
\sum_{p} \phi_{p}^{1} \otimes \epsilon\left(\phi_{p}^{2}\right)=\phi
$$

Since $\epsilon(\phi)$ is a complex number, the symbol $\otimes$ is redundant in this formula. It stands for ordinary multiplication with a complex number.

Using eqs. (2.16), (2.12) and (2.18) in turn, we find

$$
\begin{aligned}
U(\phi) \Psi_{i}^{I}|0\rangle & =\sum_{p} \Psi_{m}^{I} \tau_{m i}^{I}\left(\phi_{p}^{1}\right) U\left(\phi_{p}^{2}\right)|0\rangle \\
& =\sum_{p} \Psi_{m}^{I} \tau_{m i}^{I}\left(\phi_{p}^{1}\right)|0\rangle \epsilon\left(\phi_{p}^{2}\right) \\
& =\Psi_{m}^{I}|0\rangle \tau_{m i}^{I}(\phi)
\end{aligned}
$$

This shows that the "1-excitation states" $|I, i\rangle=\Psi_{i}^{I}|0\rangle$ transform according to the representation $\tau^{I}$. The derivation of this formula used covariance of field operators under $\mathscr{G}^{*}$, invariance (2.12) of the ground state $|0\rangle$, and property (2.17) of the co-unit. All of these will be retained in the case of more general symmetries later on.

Similarly, the transformation law of states

$$
\left|I_{1}, i_{1} \ldots i, I_{n}, i_{n}\right\rangle=\Psi_{i_{1}}^{I_{1}}, \ldots, \Psi_{i_{n}}^{I_{n}}|0\rangle
$$

is determined. Applying $U(\phi)$ to such a state, one shifts the representation operator $U(\cdot)$ through the fields one by one from left to right, using covariance property (2.16). Finally, an operator $U(\cdot)$ stands next to the ground state, so that one can convert it into a $c$-number using invariance $(2.12)$ of the ground state.

As a result one finds the well-known result that the states $\left|I_{1}, i_{1}, \ldots, I_{n}, i_{n}\right\rangle$ transform according to the tensor product

$$
\left(\tau^{I_{1}} \underline{\otimes}\left(\tau^{I_{2}} \underline{\otimes} \cdots \underline{\otimes}\left(\tau^{I_{n-1}} \underline{\otimes} \tau^{I_{n}}\right) \ldots\right)\right)
$$

of representations under the group G. The brackets in this formula are actually 
unnecessary because the tensor product is associative. But if one does not make use of this fact in the derivation, eq. (2.22) is what one gets. To generalize this formula, one observes that the tensor product of group representations can be defined in an abstract way by use of the co-product $\Delta$, as follows.

We distinguish in notation between the standard tensor product $\otimes$ of matrices, and more generally of algebras and of their elements, and the tensor product $\otimes$ of representations. If $\tau^{l}$ and $\tau^{J}$ are representations of $\mathscr{G}^{*}$ by matrices $\tau^{l}(\xi)$, etc., then the standard tensor product $\tau^{\prime} \otimes \tau^{J}(A)$ is defined for elements $A \in \mathscr{G}^{*} \otimes \mathscr{G}^{*}$ such as $A=\xi \otimes \eta$ in the ovbious way, $\left(\tau^{I} \otimes \tau^{J}\right)(\xi \otimes \eta)=\tau^{I}(\xi) \otimes \tau^{J}(\eta)$, where the right-hand side involves a tensor product of matrices. By contrast, the tensor product of representations $\left(\tau^{I} \otimes \tau^{J}\right)(\phi)$ is going to be defined for arguments $\phi \in \mathscr{G}^{*}$ through the formula

$$
\left(\tau^{l} \otimes \tau^{J}\right)(\phi)=\left(\tau^{l} \otimes \tau^{J}\right)(\Delta(\phi))=\sum_{p} \tau^{I}\left(\phi_{p}^{1}\right) \otimes \tau^{J}\left(\phi_{p}^{2}\right)
$$

Indeed, for group elements $\phi=a$,

$$
\left.\left(\tau^{l} \otimes \tau^{J}\right)(\Delta(a))\right)=\left(\tau^{\prime} \otimes \tau^{J}\right)(a \otimes a)=\tau^{l}(a) \otimes \tau^{J}(a)
$$

by eq. (2.15). Retaining definition (2.23) of the tensor product of representations for the group algebra $\mathscr{G}^{*}$, the tensor product transformation law of states continues to hold for arbitrary elements $\phi \in \mathscr{G}^{*}$.

By taking the adjoint of the transformation law (2.13) of field operators under group elements, one finds that the adjoint of a field operator $\Psi^{\prime}$ transforms according to the contragredient representation $\bar{\tau}^{I}$ of $\tau^{I}$. This generalizes to $\mathscr{G}^{*}$ in the same way as before. The contragredient representation is defined by

$$
\tilde{\tau}(\phi)={ }^{\mathrm{t}} \tau\left(\mathscr{S}^{-1}(\phi)\right)
$$

where $\mathscr{S}$ is the antipodal map. Its action on group elements is given by $\mathscr{S}(a)=a^{-1}$. This extends to an anti-automorphism of the group algebra in the obvious way, viz. $\mathscr{S}(\phi)=\int_{\mathrm{G}} \mathrm{d} a \Phi(a) a^{-1}$. The transformation law of the adjoint field operator can be written as

$$
U(\phi) \Psi_{i}^{I *}=\sum_{p} \Psi_{m}^{I *} \tilde{\tau}_{m i}\left(\phi_{p}^{2}\right) U\left(\phi_{p}^{1}\right)
$$

The interchange of $\phi_{p}^{1}$ and $\phi_{p}^{2}$ compared to the transformation law (2.16) of field operators is immaterial here. This is true because the co-product is co-commutative in the case of group algebras. Indeed, if $P$ interchanges factors, $P(a \otimes b)=b$ $\otimes a$, then $\Delta(a)=a \otimes a=P \Delta(a)$ for $a \in \mathrm{G}$, and this extend to $\mathscr{G}^{*}$.

However, if one wants to derive the transformation law of adjoints from the transformation law (2.16) of fields using abstract properties of the antipode but not 
co-commutativity of the coproduct, one gets either eq. (2.24) or eq. (2.24) with $\phi_{p}^{2}$ and $\phi_{p}^{1}$ interchanged, depending on the appropriate $*$-operation in $\mathscr{G}^{*} \otimes \mathscr{G}^{*}$. The formula (2.24) was first obtained for the quantum group algebra $\mathrm{U}_{q}\left(\mathrm{sl}_{2}\right)$ with $|q|=1$ in ref. [5].

\section{Quantum symmetry}

Abstracting from the example of a symmetry group as discussed in sect. 2, we find conditions on a symmetry algebra $\mathscr{G}^{*}$ which permit to spell out features that are commonly associated with symmetries in quantum theory:

(i) A tranformation law of states in the Hilbert space $\mathscr{H}$ which determines a unitary representation of $\mathscr{G}^{*}$ in $\mathscr{H}$;

(ii) Covariance properties of field operators and their adjoints;

(iii) Invariance of the ground state and the hamiltonian.

Locality and statistics will be discussed in sect. 4 .

Definition 3.1. (bi-*-algebra) $\mathrm{A} *$-algebra $\mathscr{G}^{*}$ with unit element $e$ is called a bi-*-algebra if a $*$-operation is specified on $\mathscr{G}^{*} \otimes \mathscr{G}^{*}$ and $\mathscr{G}^{*}$ is equipped with a co-product $\Delta$, a co-unit $\epsilon$, and an antipode $\mathscr{S}$, with properties as follows:

(1) The co-multiplication $\Delta: \mathscr{G}^{*} \mapsto \mathscr{G}^{*} \otimes \mathscr{G}^{*}$ is a $*$-homomorphism of algebras.

(2) The co-unit $\epsilon: \mathscr{G}^{*} \mapsto \mathbb{C}$ is a $*$-homomorphism of algebras subject to the extra condition

$$
(\mathrm{id} \otimes \epsilon) \Delta=\mathrm{id}=(\epsilon \otimes \mathrm{id}) \Delta .
$$

(3) The antipodal map $\mathscr{S}: \mathscr{G}^{*} \rightarrow \mathscr{G}^{*}$ is a $*$-anti-automorphism. It should obey the further conditions

$$
\begin{aligned}
& m_{r}\left(\left(\mathscr{S}^{-1} \otimes \mathrm{id} \otimes \mathrm{id}\right)(\mathrm{id} \otimes \Delta) \Delta(\phi)\right)=e \otimes \phi, \\
& m_{r}^{\prime}\left(\left(\mathrm{id} \otimes \mathscr{S}^{-1} \otimes \mathrm{id}\right)(\mathrm{id} \otimes \Delta) \Delta(\phi)\right)=\phi \otimes e,
\end{aligned}
$$

with multiplication operators defined by

$$
m_{r}(\xi \otimes \eta \otimes \zeta)=\eta \xi \otimes \zeta, \quad m_{r}^{\prime}(\xi \otimes \eta \otimes \zeta)=\zeta \otimes \eta \xi .
$$

Remark. Note that we do not require that $\Delta(e)=e \otimes e$. Instead, $\Delta(e)$ may be a nontrivial projector which commutes with all $\Delta(\xi), \xi \in \mathscr{G}^{*}$. In this case representation operators $\left(\pi^{I} \otimes \pi^{J}\right)(\xi)=\pi^{I} \otimes \pi^{J}(\Delta(\xi))$ will annihilate a proper subspace of the representation space $\mathrm{V}^{I} \otimes \mathrm{V}^{J}$ for some $I, J$ and all $\dot{\xi} \in \mathscr{G}^{*}$. This is so because $\Delta(\xi)=\Delta(e \xi)=\Delta(e) \Delta(\xi)$. In this way Drinfeld's remark [1], which suggests that minimal conformal models cannot have quasi Hopf algebras as symmetries, will be evaded (see below). 
The standard choice of the $*$-operation on $\mathscr{G}^{*} \otimes \mathscr{G}^{*}$ is

$$
(\xi \otimes \eta)^{*}=\xi^{*} \otimes \eta^{*},
$$

but it is also permissible to adopt the alternative choice

$$
(\xi \otimes \eta)^{*}=\eta^{*} \otimes \xi^{*},
$$

and to interpret condition (3.7) accordingly. The interchange of factors matches with the formula for adjoints of operators, $(A B)^{*}=B^{*} A^{*}$.

For the quantum group algebras $\mathrm{U}_{q}\left(\mathrm{sI}_{2}\right)$ with $q$ a root of unity which were proposed as symmetries of conformal field theory models, the above conditions will be fulfilled if we adopt the second choice (3.5) of $*$-operation on $\mathscr{G}^{*} \otimes \mathscr{G}^{*}$. The consequences of this choice will be discussed later on.

For the convenience of the reader we spell out the (anti)homomorphism properties. $\Delta, \mathscr{S}$ and $\epsilon$ should be linear maps. They should obey

$$
\begin{array}{cl}
\Delta(\xi \eta)=\Delta(\xi) \Delta(\eta), & \Delta\left(\xi^{*}\right)=\Delta(\xi)^{*}, \\
\epsilon(\xi \eta)=\epsilon(\xi) \epsilon(\eta), & \epsilon\left(\xi^{*}\right)=\overline{\epsilon(\xi)} \\
\mathscr{S}(\xi \eta)=\mathscr{S}(\eta) \mathscr{S}(\xi), & \mathscr{S}\left(\xi^{*}\right)=\mathscr{S}(\xi)^{*} .
\end{array}
$$

It follows that $\epsilon(e)=1$.

Definition 3.2. (Symmetry) Consider a quantum mechanical system with hamiltonian $H$ whose Hilbert space of states $\mathscr{H}$ is generated from a ground state $|0\rangle$ by field operators $\Psi_{i}^{I}(\mathbf{r}, t)$. A bi-*-algebra $\mathscr{G}^{*}$ with unit element $e$, co-product $\Delta$, co-unit $\epsilon$ and antipode $\mathscr{S}$ is called a symmetry of this system if $\mathscr{H}$ carries a unitary representation $U$ of $\mathscr{G}^{*}$, the ground state $|0\rangle$ is invariant, all representation operators $U(\phi)$ commute with the hamiltonian, and field operators transform covariantly. This means that for all $\phi \in \mathscr{G}^{*}$ we should have

$$
\begin{aligned}
U\left(\phi^{*}\right) & =U(\phi)^{*}, \\
U(\phi)|0\rangle & =|0\rangle \epsilon(\phi), \\
{[U(\phi), H] } & =0, \\
U(\phi) \Psi_{i}^{I} & =\sum_{p} \Psi_{m}^{I} \tau_{m i}^{I}\left(\phi_{p}^{1}\right) U\left(\phi_{p}^{2}\right) \\
\text { if } \quad \Delta(\phi) & =\sum_{p} \phi_{p}^{1} \otimes \phi_{p}^{2} .
\end{aligned}
$$


From covariance properties of field operators and invariance of the ground state one obtains invariance properties of Green functions in the standard way, see below.

Neither co-commutativity nor co-associativity of the co-product were required.

Definition 3.3. (co-associativity) The co-product $\Delta$ is said to be co-associative if

$$
(\text { id } \otimes \Delta) \Delta=(\Delta \otimes \text { id }) \Delta .
$$

Lemma 3.1. If the co-multiplication is co-associative, then the properties (3.2) and (3.3) are implied by the requirement

$$
m^{\prime}\left(\mathscr{S}^{-1} \otimes \mathrm{id}\right) \Delta(\phi)=m^{\prime}\left(\mathrm{id} \otimes \mathscr{S}^{-1}\right) \Delta(\phi)=\epsilon(\phi) \epsilon
$$

for all $\phi \in \mathscr{G}^{*}$. The multiplication operator $m^{\prime}$ is defined by $m^{\prime}(\xi \otimes \eta)=\eta \xi$.

Given one co-multiplication $\Delta$,

$$
\Delta(\phi)=\sum_{p} \phi_{p}^{1} \otimes \phi_{p}^{2},
$$

there exists another one:

$$
\Delta^{\prime}(\phi)=\sum_{p} \phi_{p}^{2} \otimes \phi_{p}^{1}
$$

Definition 3.4. (co-commutativity) The co-multiplication is said to be co-commutative if $\Delta=\Delta^{\prime}$.

Examples. Compact symmetry groups yield group algebras $\mathscr{G}^{*}$ with a co-multiplication that is co-associative and co-commutative. Hopf algebras $\mathscr{G}^{*}$ are examples which fulfill all our conditions except possibly those involving the $*$-operation. In a Hopf algebra, the co-multiplication is co-associative but not necessarily co-commutative. In a quasi Hopf algebra as introduced by Drinfeld the co-multiplication is not co-associative but only "quasi-co-associative". This and further properties of quasi-triangular quasi Hopf algebras which are motivated by locality will be discussed in the next sections. Later on we will introduce a further generalization which we call "weak quasi-triangular quasi Hopf algebras".

We turn to a preliminary discussion of the transformation law of the adjoint of covariant field operators. It will involve the contragredient representation.

Definition 3.5. The contragredient representation $\tilde{\tau}$ to a representation $\tau$ of $\mathscr{G} *$ is defined by

$$
\tilde{\tau}(\phi)={ }^{\mathrm{t}} \tau\left(\mathscr{S}^{-1}(\phi)\right),
$$

where $t$ stands for the transpose 
When co-multiplication is not co-commutative, there exist actually two possible co-multiplications $\Delta$ and $\Delta^{\prime}$, and therefore also two possible covariant field transformation laws, and two possible tensor products of representations $\otimes$ and $\otimes{ }^{\prime}$. If the $*$-operation on $\mathscr{G}^{*} \otimes \mathscr{G}^{*}$ is defined by eq. (3.4) then the adjoint $\bar{\psi}^{I} *$ of $\psi^{I}$ will transform like $\psi^{I}$, except that $\tilde{\tau}^{I}$ gets substituted for $\tau^{I}$. But in the other case we have:

Proposition 3.1. Suppose that the *-operation on $\mathscr{G}^{*} \otimes \mathscr{G}^{*}$ is defined by eq. (3.5). Then validity of the one covariant field transformation law (3.15) for the field $\Psi^{I}$ will imply that its adjoint $\Psi^{I *}$ will transform the other way,

$$
U(\phi) \Psi_{i}^{I *}=\sum_{p} \Psi_{m}^{I *} \tilde{\tau}_{m i}^{I}\left(\phi_{p}^{2}\right) U\left(\phi_{p}^{1}\right)
$$

Note that the role of $\phi_{p}^{1}$ and $\phi_{p}^{2}$ got interchanged compared to (3.15). This means that $\Delta$ got replaced by $\Delta^{\prime}$. The necessary computations to establish this proposition will be presented in appendix $A$.

We shall see in sect. 4 that the presence of an $R$-element in $\mathscr{G}^{*} \otimes \mathscr{G}^{*}$ will permit to define a covariant adjoint $\bar{\Psi}^{I}$ which transforms in the same way as $\Psi^{I}$, apart from the appearance of the contragredient representation $\tilde{\tau}^{I}$ in place of $\tau^{I}$.

Let us now turn to the transformation law of states

$$
\left|I_{1}, i_{1}, \cdots, I_{n}, i_{n}\right\rangle=\Psi_{i_{1}}^{I_{1}} \cdots \Psi_{i_{n}}^{I_{n}}|0\rangle
$$

It is derived in the same way as described at the end of sect. 2. This derivation makes no reference to group elements. One uses the covariance property of field operators $(3.15)$ to shift representation operators $U(\cdot)$ through field operators from left to right until they act on the ground state $|0\rangle$. Then invariance of the ground state eq. (3.12) is used to convert the operators $U(\cdot)$ into numbers. The result is stated in theorem 3.1 below, using the appropriate notion of tensor products of representations $\underline{\otimes}$.

Definition 3.6. The tensor product of representations $\underline{\otimes}$ of a bi-*-algebra is defined with the help of the co-product,

$$
\left(\tau^{I} \otimes \tau^{J}\right)(\phi)=\left(\tau^{I} \otimes \tau^{J}\right)(\Delta(\phi))
$$

The standard tensor product $\otimes$ of matrices is associative by definition. But the tensor product $\Theta$ of representations is not associative unless the co-product is co-associative. It is associative for Hopf algebras $\mathscr{G}^{*}$ (quantum group algebras), though.

Theorem 3.1. (Transformation law of states) Suppose that the bi-*-algebra $\mathscr{G}^{*}$ is a symmetry of a quantum mechanical system as described in definition 3.2. Then 
“1-excitation states" $|I, i\rangle=\Psi_{i}^{I}|0\rangle$ transform according to representation $\tau^{I}$ of $\mathscr{G}^{*}$, viz.

$$
U(\phi)|I, i\rangle=\sum_{k}|I, k\rangle \tau_{k i}^{I}(\phi)
$$

More generally, the states $\left|I_{1}, i_{1}, \cdots, I_{n}, i_{n}\right\rangle$ transform according to tensor products of representations

$$
\begin{aligned}
& U(\phi)\left|I_{1}, i_{1}, \ldots, I_{n}, i_{n}\right\rangle \\
& \quad=\left|I_{1}, k_{1}, \ldots, I_{n}, k_{n}\right\rangle\left(\tau^{I_{1}} \underline{\otimes} \ldots\left(\tau^{I_{n-1}} \underline{\otimes} \tau^{I_{n}}\right) \ldots\right)_{k_{1} \ldots k_{n}, i_{1} \ldots i_{n}}(\phi) .
\end{aligned}
$$

Summation over $k_{1} \cdots k_{\mathrm{n}}$ is understood.

The representations $\tau^{I}$ furnish linear functions $\tau_{i k}^{I}$ on $\mathscr{G}^{*}$. Such linear functions may be regarded as elements of the dual $\mathscr{G}$ of $\mathscr{G}^{*}$. The co-product $\Delta$ in $\mathscr{G}^{*}$ yields a product $\cdot$ in $\mathscr{G}$ such that

$$
\left(\tau^{I} \otimes \tau^{J}\right)_{i j, k l}(\phi)=\left(\tau_{i k}^{I} \cdot \tau_{j l}^{J}\right)(\phi)
$$

The details of this construction shall be recalled in the sect. 8 . In general this product is neither commutative nor associative. In this new notation, the transformation law (3.25) can be written in the equivalent form

$$
\begin{aligned}
& U(\phi)\left|I_{1}, i_{1}, \ldots, I_{n}, i_{n}\right\rangle \\
& \quad=\left|I_{1}, k_{1}, \ldots, I_{n}, k_{n}\right\rangle\left(\tau_{k_{1} i_{1}}^{I_{1}} \cdot \ldots \cdot\left(\tau_{k_{n-1} i_{n-1}}^{I_{n-1}} \cdot \tau_{k_{n} i_{n}}^{I_{n}}\right) \ldots\right)(\phi) .
\end{aligned}
$$

Let us finally turn to the invariance properties of Green functions. Taking the adjoint of the eq. (3.12) which expresses invariance of the ground state, and using the unitarity conditions on $U$ and $\epsilon$ we find that

$$
\langle 0| U(\phi)=\epsilon(\phi)\langle 0|
$$

It follows that

$$
\epsilon(\phi)\left\langle 0\left|\Psi_{i_{1}}^{I_{1}} \ldots \Psi_{i_{n}}^{I_{n}}\right| 0\right\rangle=\left\langle 0\left|U(\phi) \Psi_{i_{1}}^{I_{1}} \ldots \Psi_{i_{n}}^{I_{n}}\right| 0\right\rangle .
$$

Inserting the transformation law of states derived before, one obtains the invariance property stated in the following theorem.

Theorem 3.2. (Invariance properties of correlation functions) Under the same hypotheses as in theorem 3.1, correlation functions are invariant in the sense that

$$
\begin{aligned}
& \epsilon(\phi)\left\langle 0\left|\Psi_{i_{1}}^{I_{1}} \ldots \Psi_{i_{n}}^{I_{n}}\right| 0\right\rangle \\
& \quad=\left\langle 0\left|\Psi_{m_{1}}^{I_{1}} \ldots \Psi_{m_{n}}^{I_{n}}\right| 0\right\rangle\left(\tau_{m_{1} i_{1}}^{I_{1}} \ldots \cdot\left(\tau_{m_{n-I^{i_{n}-1}}}^{I_{n-1}} \cdot \tau_{m_{n} i_{n}}^{I_{n}}\right) \ldots\right)(\phi) .
\end{aligned}
$$


This involves a tensor product of representations, written in the compact notation which involves the noncommutative product $\cdot$ in $\mathscr{G}$, see eq. (3.26) above.

In special cases it is known that the most general Green function which satisfies these invariance properties is a weighted sum of products of suitably defined Clebsch-Gordan coefficients for tensor products of irreducible representations, much as in the case of symmetry groups. This is true for $\mathrm{U}_{q}\left(\mathrm{sl}_{2}\right)$ for instance when $q$ is not a root of unity [24]. In general one will need information about the decomposition of the representation $U$ into irreducibles to obtain such a representation. This is why one prefers semisimple symmetry algebras whose representations are all fully reducible.

\section{Locality}

Local Fermi or Bose fields obey anticommutation or commutation relations

$$
\Psi_{i}^{I}\left(r_{1}, t\right) \Psi_{j}^{J}\left(r_{2}, t\right)= \pm \Psi_{j}^{J}\left(r_{2}, t\right) \Psi_{i}^{I}\left(r_{1}, t\right) \quad \text { if } r_{1} \neq r_{2}
$$

In the case of a Lorentz covariant theory this has to hold in an arbitrary reference frame and implies local (anti-)commutation relations at arbitrary spacelike distances.

It has been discussed in detail in the literature that there are more general possibilities in 1 and 2 space dimensions [2,26,27]. When one wants to interchange positions of two particles or arguments of wave functions $\boldsymbol{r}_{1}$ and $\boldsymbol{r}_{2} \neq \boldsymbol{r}_{1}$ one may encounter two different situations which we distinguish symbolically as $\boldsymbol{r}_{2}>\boldsymbol{r}_{1}$ and $r_{2}<r_{1}{ }^{*}$. These two different situations may require two different interchanging operations $\sigma_{1}$ and $\sigma_{1}^{-1}$. More generally, when there are $N$ particles there may be two operations $\sigma_{i}$ and $\sigma_{i}^{-1}$ which interchange particle $i$ and $i+1$. These operations must satisfy the Artin relations

$$
\sigma_{i} \sigma_{k}=\sigma_{k} \sigma_{i} \text { if }|k-i| \geqslant 2, \quad \sigma_{i} \sigma_{i+1} \sigma_{i}=\sigma_{i+1} \sigma_{i} \sigma_{i+1}
$$

As a result, $\sigma_{1} \ldots \sigma_{n-1}$ generate the braid group $\mathrm{B}_{n}$ on $n$ threads. Using the sraphical notation of fig. 1a, a general element

$$
b=\sigma_{i_{1}}^{ \pm 1} \ldots \sigma_{i_{k}}^{ \pm 1}
$$

an be represented by a braid, as in fig. $1 \mathrm{~b}$, and multiplication of group elements :orresponds with juxtaposition of braids. Braids which can be continuously de-

In 1 space dimensions $r_{2}>r_{1}$ if the real coordinate $r_{2}$ is bigger than $r_{1}$. In 2 space dimensions wave functions may be multivalued, i.e. have cuts, and the two possibilities differ in the relative positions to the cut (see ref. [27]). 


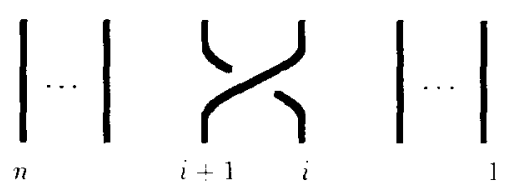

$$
\sigma_{i}
$$

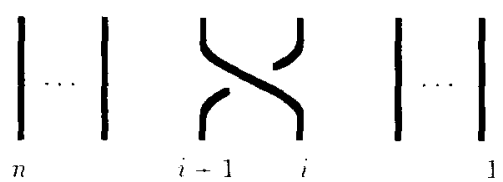

(a)

$$
\sigma_{i}^{-1}
$$

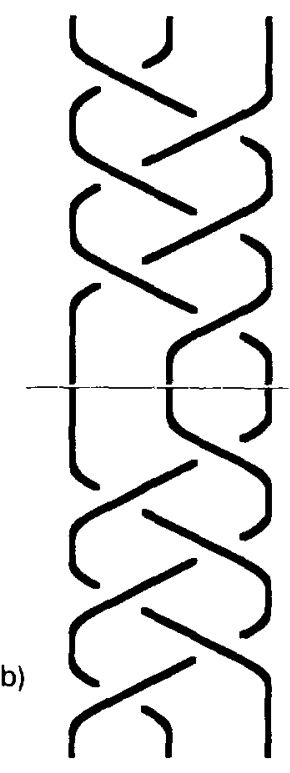
(b) A braid multiplied with its inverse.

formed into each other (holding ends fixed) with the help of operations as in fig. 2 are regarded to be the same. The possible deformations express the Artin relations and the further relation $\sigma_{i}^{-1} \sigma_{i}=\sigma_{i} \sigma_{i}^{-1}=1$. As a result, the braid shown in fig. $1 \mathrm{~b}$
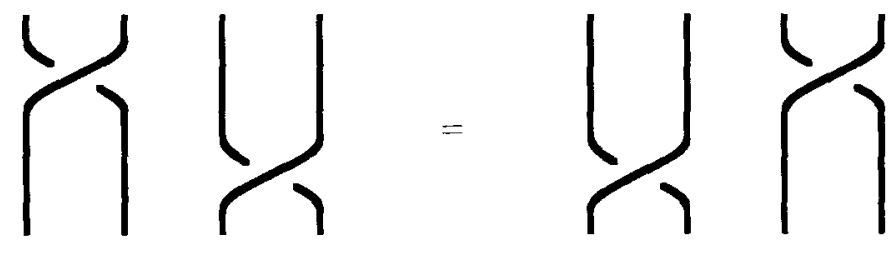

$$
\sigma_{3} \sigma_{1}=\sigma_{1} \sigma_{3}
$$
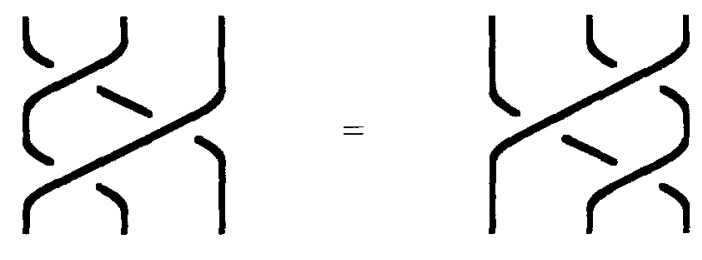

$$
\sigma_{2} \sigma_{1} \sigma_{2}=\sigma_{1} \sigma_{2} \sigma_{1}
$$

Fig. 2. Deformations of braids. 
is the product of a braid $b$ (above the dashed line) with its inverse, and is thus equal to the identity braid (parallel lines). Every braid determines a permutation in the obvious way, but $\sigma_{i}$ and $\sigma_{i}^{-1}$ determine the same permutation $i \mapsto i+1$. Therefore the permutation group $\mathscr{S}_{n}$ is a factor group of the braid group $\mathrm{B}_{n}$. Bose-Fermi statistics can be regarded as a special case of braid group statistics with $\sigma_{i}^{-1}=\sigma_{i}$.

Fröhlich proposed that field operators should obey local braid relations of the following form in the case of braid group statistics:

$$
\Psi_{i}^{I}\left(\boldsymbol{r}_{1}, t\right) \Psi_{j}^{J}\left(\boldsymbol{r}_{2}, t\right)=\Psi_{m}^{J}\left(\boldsymbol{r}_{2}, t\right) \Psi_{n}^{I}\left(\boldsymbol{r}_{1}, t\right) \mathscr{R}_{n m, i j}^{I J>} \text { if } \boldsymbol{r}_{2}>\boldsymbol{r}_{1}
$$

and a similar relation involving $\mathscr{R}^{I J}<$ if $\boldsymbol{r}_{2}<\boldsymbol{r}_{1}$. The two matrices are related as follows. Denoting by $\hat{\mathscr{R}}$ the matrix obtained from $\mathscr{R}$ by interchange of the first two indices $n, m$, the two matrices $\mathscr{R}^{>}$and $\mathscr{R}^{<}$are related by

$$
\hat{\mathscr{K}}^{I J<}=\left(\hat{\mathscr{R}}^{J I>}\right)^{-1}
$$

The local braid relations for $\boldsymbol{r}_{2}<\boldsymbol{r}_{1}$ follow from those for $\boldsymbol{r}_{2}>\boldsymbol{r}_{1}$ by inversion using (4.4) and will therefore not lead to independent consistency conditions.

Fröhlich proposed that local braid relations (4.3) should hold with a numerical matrix $\mathscr{R}$. In this case, consistency requires that matrices $\mathscr{R}$ should satisfy the Yang-Baxter equations. This assures that $\mathscr{R}$ yields a representation of the braid group. The Yang-Baxter equations are a translation of the Artin relations (see ref. [2]).

As we shall see, quasi Hopf symmetry leads in a natural way to $\mathscr{R}$-matrices which are representation operators of certain elements of $\mathscr{G}^{*}$. Only in the special case of Hopf symmetry (quantum groups) can they be expected to be numerical matrices.

It is nevertheless instructive to examine first the conditions that come from the requirement that both sides of the local braid relations transform in the same way under the symmetry, assuming that $\mathscr{R}$ is a numerical matrix. The calculation is presented in appendix $B$. The result is as follows.

Suppose that there exists an element $R$ in the algebra,

$$
R=\sum_{a} r_{a}^{1} \otimes r_{a}^{2} \in \mathscr{G}^{*} \otimes \mathscr{G}^{*}
$$

such that

$$
\mathscr{R}_{t u, s r}^{I J}=c\left(\tau^{I} \otimes \tau^{J}\right)_{t u, s r}(R)=c \sum_{a} \tau_{t s}^{I}\left(r_{a}^{1}\right) \tau_{u r}^{J}\left(r_{a}^{2}\right)
$$

where $c$ is a numerical phase factor. Then the following condition is sufficient:

$$
(R \otimes e) \cdot(\mathrm{id} \otimes \Delta) \Delta(\phi)=\left(P_{12}(\mathrm{id} \otimes \Delta) \Delta(\phi)\right) \cdot(R \otimes e),
$$


where $P_{12}\left(a_{1} \otimes a_{2} \otimes a_{3}\right)=a_{2} \otimes a_{1} \otimes a_{3}$ and we wrote $\cdot$ for the product in $\mathscr{G}^{*} \otimes \mathscr{G}^{*}$ $\otimes \mathscr{G}^{*}$ for the sake of clarity.

In a Hopf algebra, co-multiplication is co-associative, so that eq. (3.17) holds. Then eq. (4.7) is fulfilled if

$$
(R \otimes e) \cdot(\Delta \otimes \mathrm{id})(\xi \otimes \eta)=\left(P_{12}(\Delta \otimes \mathrm{id})(\xi \otimes \eta)\right)(R \otimes e) \text { for all } \xi \in \mathscr{G}^{*} .
$$

This is equivalent to

$$
R \Delta(\xi)=\Delta^{\prime}(\xi) R \text { for all } \xi \in \mathscr{G}^{*},
$$

where $\Delta^{\prime}$ differs from $\Delta$ by the interchange of factors [cf. eq. (3.20)].

Hopf algebras with an element $R \in \mathscr{G}^{*} \otimes \mathscr{G}^{*}$ satisfying eq. (4.8) are called quasi-triangular, or quantum group algebras. $\mathrm{U}_{q}\left(\mathrm{sl}_{2}\right)$ are examples.

In conclusion we see that quantum group symmetry is consistent with local braid relations with a numerical $\mathscr{R}$-matrix that satisfies the Yang-Baxter equations. It is a special case of quantum symmetry in which the co-multiplication is co-associative.

By Einstein causality, observables have to satisfy ordinary Bose commutation relations. A nontrivial transformation law under a quantum symmetry which does not come from a group will typically be consistent with an appropriate form of local braid relations but not in general with Bose commutation relations. It follows that observables should be invariant under quantum symmetries. (Global) symmetries which leave the observables invariant are known as gauge symmetries (of first kind). They occur in theories with superselection sectors [5,24,26,28]. Fields with a nontrivial transformation law under such a gauge symmetry make transitions between different superselection sectors.

\section{Quasi Hopf symmetry}

Definition 5.1. (quasi-co-associativity) The co-multiplication $\Delta$ of $\mathscr{G}^{*}$ is quasico-associative if there exists an invertible element $\varphi \in \mathscr{G}^{*} \otimes \mathscr{G}^{*} \otimes \mathscr{G}^{*}$ such that

$$
\varphi(\Delta \otimes \text { id }) \Delta(\xi)=(\text { id } \otimes \Delta) \Delta(\xi) \varphi \text { for all } \xi \in \mathscr{G}^{*} \text {. }
$$

If the co-multiplication is not co-associative, then the tensor product of representations of $\mathscr{G}^{*}$ is not associative in general. However, quasi-associativity of co-multiplication implies that representations

$$
\left(\pi^{1} \otimes \pi^{2}\right) \otimes \pi^{3} \text { and } \pi^{1} \otimes\left(\pi^{2} \otimes \pi^{3}\right)
$$

are equivalent. 
Quasi-triangular quasi Hopf algebras as introduced by Drinfeld [1] are bi-algebras in which the co-multiplication is quasi co-associative. They possess a co-unit, antipode and $R$-element $R \in \mathscr{G}^{*} \otimes \mathscr{G}^{*}$ which satisfy certain properties. We shall see that symmetry under a quasi-triangular Hopf algebra is consistent with an appropriate form of local braid relations.

If co-multiplication is no co-associative, then the covariant field transformation law (3.15) does not imply a covariant transformation law of products of fields. But co-associativity helps to define a new product of field operators which is covariant.

Theorem 5.1. (covariant product of field operators) Suppose that the bi-*-algebra $\mathscr{G}^{*}$ is a symmetry of a quantum mechanical system in the sense of definition 3.2 , and that there exists

$$
\varphi=\sum_{\sigma} \varphi_{\sigma}^{1} \otimes \varphi_{\sigma}^{2} \otimes \varphi_{\sigma}^{3}, \quad \varphi_{\sigma}^{i} \in \mathscr{G}^{*},
$$

which satisfies eq. (5.1). Define

$$
\left(\Psi^{I} \times \Psi^{J}\right)_{i j}=\sum_{\sigma} \Psi_{m}^{I} \Psi_{n}^{J} \tau_{m i}^{I}\left(\varphi_{\sigma}^{1}\right) \tau_{n j}^{J}\left(\varphi_{\sigma}^{2}\right) U\left(\varphi_{\sigma}^{3}\right)
$$

Then $\Psi^{I} \times \Psi^{J}$ transforms according to the tensor product $\tau^{I} \otimes \tau^{J}$ of representations of $\mathcal{G}^{*}$ in the sense that it obeys transformation law (3.15).

This is verified by straightforward computation. Note that invertibility of $\varphi$ (which we wish to relax later on) is not assumed in theorem 5.1.

The covariant product $x$ is not associative in general. But a straightforward computation establishes the following theorem.

Theorem 5.2. (quasi-associativity of the covariant product of fields) If $\varphi$ satisfies Drinfeld's relation

$$
(\mathrm{id} \otimes \mathrm{id} \otimes \Delta)(\varphi)(\Delta \otimes \mathrm{id} \otimes \mathrm{id})(\varphi)=(e \otimes \varphi)(\mathrm{id} \otimes \Delta \otimes \mathrm{id})(\varphi)(\varphi \otimes e)
$$

then the product $x$ is quasi-associative in the sense that

$$
\left(\left(\Psi^{I} \times \Psi^{J}\right) \times \Psi^{K}\right)_{i j k}=\left(\Psi^{I} \times\left(\Psi^{J} \times \Psi^{K}\right)\right)_{i^{\prime} j^{\prime} k^{\prime}}\left(\tau^{I} \otimes \tau^{J} \otimes \tau^{K}\right)_{i^{\prime} j^{\prime} k^{\prime}, i j k}(\varphi) .
$$

Explicitly

$$
\left(\tau^{I} \otimes \tau^{J} \otimes \tau^{K}\right)_{i^{\prime} j^{\prime} k^{\prime}, i j k}(\varphi)=\sum_{\sigma} \tau_{i^{\prime} i}^{I}\left(\varphi_{\sigma}^{1}\right) \tau_{j^{\prime} j}^{\prime}\left(\varphi_{\sigma}^{2}\right) \tau_{k^{\prime} k}^{K}\left(\varphi_{\sigma}^{3}\right)
$$

Next we turn to the discussion of local braid relations for field operators. It is consistent to demand that

$$
\left(\Psi^{\prime} \times \Psi^{J}\right)_{i j}=\left(\Psi^{J} \times \Psi^{l}\right)_{j^{\prime} i^{\prime}} \tilde{\mathscr{R}}_{i^{\prime} j^{\prime}, i j}
$$


if the arguments of the field operators, which we neglect to write, satisfy conditions as in eq. (4.3). Because of the covariance properties of field operators, both sides will transform in the same way if there exists an element $R \in \mathscr{G}^{*} \otimes \mathscr{G}^{*}$ satisfying the relation

$$
\Delta^{\prime}(\eta) R=R \Delta(\eta) \text { for all } \eta \in \mathscr{G}^{*}
$$

and $\tilde{\mathscr{R}}$ in eq. (5.7) is a numerical matrix which is obtained from $R$, up to a phase factor $c$ which may depend on $I$ and $J$, as

$$
\overrightarrow{\mathscr{R}}=c\left(\tau^{I} \otimes \tau^{J}\right)(R)
$$

If $R=\sum r_{a}^{1} \otimes r_{a}^{2}$ then this reads explicitly as follows:

$$
\tilde{\mathscr{R}}_{i^{\prime} j^{\prime}, i j}=c^{I J} \sum_{a} \tau_{i^{\prime} i}^{l}\left(r_{a}^{1}\right) \tau_{j^{\prime} j}^{J}\left(r_{a}^{2}\right)
$$

We neglect to write superscripts $I J>$ on $\tilde{\mathscr{R}}$.

Later on we shall wish to relax invertibility of $\varphi$. But for now, we adopt the hypothesis that $\varphi$ has an inverse which we write as

$$
\varphi^{-1}=\sum_{\sigma} \xi_{\sigma}^{1} \otimes \xi_{\sigma}^{2} \otimes \xi_{\sigma}^{3} .
$$

It follows that ordinary products of field operators can be recovered from covariant ones,

$$
\Psi_{i}^{I} \Psi_{j}^{J}=\sum_{\sigma}\left(\Psi^{I} \times \Psi^{J}\right)_{i^{\prime} j^{\prime}} \tau_{i^{\prime} i}^{I}\left(\xi_{\sigma}^{l}\right) \tau_{j^{\prime} j}^{J}\left(\xi_{\sigma}^{2}\right) U\left(\xi_{\sigma}^{3}\right)
$$

Inserting the local braid relations in the form (5.7) and using the definition of the covariant product once again, one finds that Fröhlich's local braid relations (4.3) hold. But the $R$-matrix elements $\mathscr{R}_{i^{\prime} j^{\prime}, i j}$ are not numbers any more but operators in Hilbert space,

$$
\mathscr{R}_{i^{\prime} j^{\prime}, i j}=c^{I J} \sum_{\tau, a, \sigma} \tau_{i^{\prime} i}^{I}\left(\varphi_{\sigma}^{2} r_{a}^{1} \xi_{\tau}^{1}\right) \tau_{j^{\prime} j}^{J}\left(\varphi_{\sigma}^{1} r_{a}^{2} \xi_{\tau}^{2}\right) U\left(\varphi_{\sigma}^{3} \xi_{\tau}^{3}\right)
$$

This is a linear combination of representation operators $U\left(\varphi_{\sigma}^{3} \xi_{\tau}^{3}\right)$ and is therefore equal to a representation operator $U(\eta)$ of some element $\eta \in \mathscr{G}^{*}$. Expression (5.13) involves the element

$$
\begin{gathered}
\sum_{\tau, a, \sigma} \varphi_{\sigma}^{2} r_{a}^{1} \xi_{\tau}^{1} \otimes \varphi_{\sigma}^{1} r_{a}^{2} \xi_{\tau}^{2} \otimes \varphi_{\sigma}^{3} \xi_{\tau}^{3} \\
=\varphi_{213}(R \otimes e) \varphi^{-1} \in \mathscr{G}^{*} \otimes \mathscr{G}^{*} \otimes \mathscr{G}^{*} .
\end{gathered}
$$


Here and in the following we use the notation

$$
\varphi_{s(1) s(2) s(3)}=\sum_{\sigma} \varphi_{\sigma}^{s^{-1}(1)} \otimes \varphi_{\sigma}^{s^{-1}(2)} \otimes \varphi_{\sigma}^{s^{-1}(3)}
$$

for any permutation $s$ of 1,2, 3 if $\varphi$ is given by eq. (5.3). The same notation will be used for $\xi=\varphi^{-1}$.

Consistency requires that the matrix $\tilde{\mathscr{R}}$ satisfies constraints which come from the possibility of interchanging triples of fields in two different ways, leading to the same result. We write $\Psi^{1}$ for $\Psi^{I_{1}}\left(\boldsymbol{r}_{1}, t\right)$ and $\tau^{1}$ for $\tau^{I_{1}}$. The constraint is that the following diagram must be commutative.

$$
\begin{array}{lr}
\left(\Psi^{1} \times \Psi^{2}\right) \times \Psi^{3} \mapsto\left(\Psi^{2} \times \Psi^{1}\right) \times \Psi^{3} \mapsto \Psi^{2} \times\left(\Psi^{1} \times \Psi^{3}\right) \mapsto \Psi^{2} \times\left(\Psi^{3} \times \Psi^{1}\right) \mapsto & \left(\Psi^{2} \times \Psi^{3}\right) \times \Psi^{1} \\
& \downarrow \\
\Psi^{1} \times\left(\Psi^{2} \times \Psi^{3}\right) & \left(\Psi^{3} \times \Psi^{2}\right) \times \Psi^{1} \\
\downarrow & \downarrow \\
\Psi^{1} \times\left(\Psi^{3} \times \Psi^{2}\right) \mapsto\left(\Psi^{1} \times \Psi^{3}\right) \times \Psi^{2} \rightarrow\left(\Psi^{3} \times \Psi^{1}\right) \times \Psi^{2} \rightarrow \Psi^{3} \times\left(\Psi^{1} \times \Psi^{2}\right) \mapsto \Psi^{3} \times\left(\Psi^{2} \times \Psi^{1}\right)
\end{array}
$$

Let us suppose that $r_{1}<r_{2}<r_{3}$. The operations represented by arrows may then be performed by use of the local braid relations (5.7) and quasi-associativity (5.6) of the product $\times$. Using eq. (5.9) one sees that the resulting relation for $\mathscr{R}$ is fulfilled if $R \in \mathscr{G}^{*} \otimes \mathscr{G}^{*}$ satisfies Drinfeld's quasi Yang-Baxter equations. We use the standard notation

$$
R_{13}=\sum r_{a}^{1} \otimes 1 \otimes r_{a}^{2} \in \mathscr{G}^{*} \otimes \mathscr{G}^{*} \otimes \mathscr{G}^{*},
$$

etc. $\left(e=\right.$ unit element in $\left.\mathscr{G}^{*}\right)$. The quasi Yang-Baxter equations read

$$
R_{12} \varphi_{312} R_{13} \varphi_{132}^{-1} R_{23} \varphi=\varphi_{321} R_{23} \varphi_{231}^{-1} R_{13} \varphi_{213} R_{12}
$$

In the case that the co-product is associative, $\varphi$ is equal to the identity and the quasi Yang-Baxter equation becomes the ordinary Yang-Baxter equation

$$
R_{12} R_{13} R_{23}=R_{23} R_{13} R_{12}
$$

It is known to follow from general principles that the Hilbert space of physical states carries a unitary representation of the braid group [26]. The quasi Yang-Baxter equations are a reflection of this fact. Consider states which are created from the ground state $|0\rangle$ by application of a covariant product $\Psi^{1} \times \cdots \times \Psi^{N}$ of field operators $\Psi^{1} \ldots \Psi^{N}$ which depend on arguments $r_{1}, \ldots, r_{N}$. Suppose that $r_{1}<r_{2}$ $<\ldots<\boldsymbol{r}_{N}$, for instance. Complete specification of the multiple covariant product requires putting brackets, but we may use quasi-associativity to rearrange the brackets. Suppose this is done in a suitable way so that $\left(\Psi^{i} \times \Psi^{i+1}\right)$ appears. Then we may define the action of $\sigma_{i}$ on the state by interchanging $\Psi^{i}$ and $\Psi^{i+1}$. (This is 
true if $\mathbf{r}_{i}<\mathbf{r}_{i+1}$. In the other case this operation is $\sigma_{i}^{-1}$.) This action of $\sigma_{i}$ 's must satisfy the second Artin relation (4.2). Commutativity of the diagram (5.17) assures this.

Finally one would like to have validity of local braid relations also for composite fields. Therefore it is natural to require that braid relations $(5.7)$ are valid when $\Psi^{\prime}$ is a covariant product of $\Psi^{I_{1}} \times \Psi^{I_{2}}$ of field operators, with a braid matrix (5.9) involving representation $\tau^{I} \equiv \tau^{I_{1}} \otimes \tau^{I_{2}}$. Thus we should have that

$$
\left(\Psi^{1} \times\left(\Psi^{2} \times \Psi^{3}\right)\right)_{i j k}=\left(\left(\Psi^{2} \times \Psi^{3}\right) \times \Psi^{1}\right)_{j^{\prime} k^{\prime} i^{\prime}} \tilde{\mathscr{R}}_{i^{\prime} j^{\prime} k^{\prime}, i j k}
$$

with

$$
\overline{\mathscr{R}}=c \sum_{a} \tau^{1}\left(r_{a}^{1}\right) \otimes\left(\tau^{2} \otimes \tau^{3}\right)\left(r_{a}^{2}\right)
$$

where $c$ is a phase factor. Similarly, consideration of composite $\Psi^{J}$ leads to

$$
\left(\left(\Psi^{1} \times \Psi^{2}\right) \times \Psi^{3}\right)_{i j k}=\left(\Psi^{3} \times\left(\Psi^{1} \times \Psi^{2}\right)\right)_{j^{\prime} k^{\prime} i^{\prime}} \overline{\mathscr{R}}_{i^{\prime} j^{\prime} k^{\prime}, i j k}
$$

with

$$
\tilde{\mathscr{R}}^{\prime}=c^{\prime} \sum_{a}\left(\tau^{\prime} \otimes \tau^{2}\right)\left(r_{a}^{1}\right) \otimes \tau^{3}\left(r_{a}^{2}\right)
$$

The result of the exchange (5.20) can be determined from the simple braid relation (5.7) and quasi-associativity as shown in the following diagram:

$$
\begin{array}{ccc}
\left(\Psi^{1} \times \Psi^{2}\right) \times \Psi^{3} & \mapsto & \Psi^{3} \times\left(\Psi_{\uparrow}^{1} \times \Psi^{2}\right) \\
\Psi^{1} \times\left(\Psi^{2} \times \Psi^{3}\right) \mapsto \Psi^{1} \times\left(\Psi^{3} \times \Psi^{2}\right) \mapsto\left(\Psi^{1} \times \Psi^{3}\right) \times \Psi^{2} \mapsto & \left(\Psi^{3} \times \Psi^{1}\right) \times \Psi^{2} .
\end{array}
$$

This leads to the same result as (5.20) and (5.21) if

$$
(\mathrm{id} \otimes \Delta)(R)=\varphi_{231}^{-1} R_{13} \varphi_{213} R_{12} \varphi^{-1} .
$$

Similarly, eqs. (5.22) and (5.23) are valid if

$$
(\Delta \otimes \mathrm{id})(R)=\varphi_{312} R_{13} \varphi_{132}^{-1} R_{23} \varphi \text {. }
$$

These two relations are true by definition in a quasi-triangular quasi Hopf algebra [1].

Conversely we have now motivated all the relations (5.5), (4.8), (5.18), (5.25) and (5.26) between $R, \varphi$ and $\Delta$, which are valid in a quasi-triangular quasi Hopf algebra, from natural postulates on properties of quantum fields (The quasi 
Yang-Baxter equations (5.18) are actually consequences of eqs. (5.25) and (5.26) [1].) Invertibility of $\varphi$ and $R$ could be relaxed as we shall see in sect. 6 .

There is also a co-unit and an antipode in a quasi Hopf algebra. They serve to state invariance of the ground state and the transformation law of adjoints of field operators as was explained earlier.

\section{Weak quasi Hopf algebras}

We would like to admit the possibility that

$$
\Delta(e) \neq e \otimes e .
$$

Since $e^{2}=e=e^{*}$ and $\Delta$ is supposed to be a $*$-morphism, $\Delta(e)$ is a projector, i.e. self-adjoint and idempotent. If it is a nontrivial projector, then for some $I, J$ the range and co-kernel of the tensor product representation $\pi^{I} \otimes \pi^{J}$ will have dimension less than the product of the dimensions of the representations $\pi^{I}$ and $\pi^{J}$. This is so because $e \xi=\xi$ implies

$$
\begin{aligned}
\pi^{I} \otimes \pi^{J}(\xi) & =\pi^{I} \otimes \pi^{J}(\Delta(\xi)) \\
& =P_{I J} \pi^{I} \underline{\otimes} \pi^{J}(\xi)=\pi^{I} \otimes \pi^{J}(\xi) P_{I J}, \\
P_{I J} & =\left(\pi^{I} \otimes \pi^{J}\right)(\Delta(e)) .
\end{aligned}
$$

In this way we can hope to accommodate the truncated tensor products of representations of $\mathrm{U}_{q}\left(\mathrm{sl}_{2}\right)$ which appear in conformal field theory - see sect. 5. To accommodate this possibility, we must weaken the requirement that $R$ and the associator $\varphi$ both have inverses. Otherwise, the right-hand side of eq. (5.25) has an inverse and the left-hand side does not.

Let us recall that an element $\varphi$ of an algebra is said to possess a quasi-inverse $\xi$ if $\varphi \xi \varphi=\varphi$.

Definition 6.1. (weak quasitriangular quasi Hopf $*$-algebra) A bi-*-algebra $\mathscr{G}^{*}$ with unit $e$, co-product $\Delta$, co-unit $\epsilon$ and antipode $\mathscr{S}$ is called a weak quasi-triangular quasi Hopf $*$-algebra if there exist elements $\varphi \in \mathscr{G}^{*} \otimes \mathscr{G}^{*} \otimes \mathscr{G}^{*}$ and $R \in$ $\mathscr{G}^{*} \otimes \mathscr{G}^{*}$ which possess quasi-inverses, denoted by $\varphi^{-1}$ and $R^{-1}$, such that

$$
\begin{aligned}
& \varphi \varphi^{-1}=(\mathrm{id} \otimes \Delta) \Delta(e) \\
& \varphi^{-1} \varphi=(\Delta \otimes \mathrm{id}) \Delta(e), \\
& R R^{-1}=\Delta^{\prime}(e) \\
& R^{-1} R=\Delta(e),
\end{aligned}
$$

$(\mathrm{id} \otimes \mathrm{id} \otimes \epsilon)(\varphi)=(\mathrm{id} \otimes \epsilon \otimes \mathrm{id})(\varphi)=(\epsilon \otimes \mathrm{id} \otimes \mathrm{id})(\varphi)=\Delta(e)$, 
and which satisfy Drinfeld's relations (4.8), (5.1), (5.5), (5.18), (5.25) and (5.26) between co-unit $\epsilon, R, \varphi$ and $\Delta$. In particular, $R \Delta(\xi)=\Delta^{\prime}(\xi) R$ for all $\xi \in \mathscr{G}^{*}$.

When the properties involving *-operations are not assumed, we speak of a weak quasi-triangular quasi Hopf algebra.

Convention. When a quasi-triangular quasi Hopf algebra $\mathscr{G}^{*}$ is mentioned, it is always understood that the symbols $\Delta, \epsilon, R, R^{-1}, \varphi, \varphi^{-1}$ have the same meaning as in definition 6.1.

The existence of a quasi-inverse permits to deduce from eq. (5.1) that

$$
\begin{aligned}
\varphi(\Delta \otimes \mathrm{id}) \Delta(\xi) \varphi^{-1} & =(\mathrm{id} \otimes \Delta) \Delta(\xi) \\
(\Delta \otimes \mathrm{id}) \Delta(\xi) & =\varphi^{-1}(\mathrm{id} \otimes \Delta) \Delta(\xi) \varphi
\end{aligned}
$$

for all $\xi \in \mathscr{G}^{*}$. Therefore we have the following theorem.

Theorem 6.1. (quasi-associativity of the tensor product of representations) The tensor product representations

$$
\left(\pi^{I} \otimes \pi^{J}\right) \underline{\otimes} \pi^{K} \text { and } \pi^{I} \underline{\otimes}\left(\pi^{J} \underline{\otimes} \pi^{K}\right)
$$

of a weak quasi-triangular quasi Hopf $*$-algebra are equivalent.

There are various consistency checks which one can make to get familiar with the setup. For instance, one may multiply eq. (5.26) for $(\Delta \otimes \mathrm{id})(R)$ with $(\Delta \otimes$ $\mathrm{id})(\Delta(e))$ from the right. On both sides this amounts to doing nothing. One may also push the factor $(\Delta \otimes \mathrm{id})(\Delta(e))$ through the operators on the right-hand side from right to left. In each step it gets converted into another projector which matches with the range of the preceding operator and with the co-kernel of the next one.

We will not make an attempt to derive property (6.9) from the other properties of a weak quasi-triangular quasi Hopf algebra, but we explain why it should hold. The co-unit defines the trivial one-dimensional representation, $\xi \mapsto \epsilon(\xi)$. Identity (3.1) assures that tensoring with this one-dimensional representation amounts to doing nothing. Therefore the map which implements the equivalence of the two triple products of representations (6.12) should be trivial when one of the representations $\pi^{I}, \pi^{J}, \pi^{K}$ is the trivial one-dimensional one. Identity (6.9) assures that.

We would like to argue that the conclusions of sect. 5 remain unchanged when we weaken invertibility requirements in the manner described above, and that we should therefore regard weak quasi-triangular quasi Hopf algebras as possible symmetries just as well.

There is only one point which needs discussion. We used the existence of an inverse of $\varphi$ to recover local braid relations involving ordinary products of fields from the local braid relation (5.7) for covariant products. 
Let $\Delta(e)=\sum e_{p}^{1} \otimes e_{p}^{2}$. Given the fields $\Psi^{I}$, we may define new fields $\tilde{\Psi}^{I}$ by

$$
\tilde{\Psi}_{i}^{I}=\left(\Psi^{I}\left(\tau^{I} \otimes U\right)(\Delta(e))\right)_{i} \equiv \sum_{p} \Psi_{j}^{l} \tau_{j i}^{I}\left(e_{p}^{1}\right) U\left(e_{p}^{2}\right)
$$

Using the field transformation law, property (3.1) of the co-unit, and $U(e)=1$, it is straightforward to verify by induction that

$$
\tilde{\Psi}_{i_{1}}^{I_{1}} \ldots \tilde{\Psi}_{i_{n}}^{I_{n}}|0\rangle=U(e) \Psi_{i_{1}}^{I_{1}} \ldots \Psi_{i_{n}}^{I_{n}}|0\rangle=\Psi_{i_{1}}^{I_{1}} \ldots \Psi_{i_{n}}^{I_{n}}|0\rangle
$$

But the Hilbert space of physical states is spanned by vectors of the form $\Psi_{i_{1}}^{I_{1}} \ldots \Psi_{i_{n}}^{I_{n}}|0\rangle$ by hypothesis. As operators in this Hilbert space, $\tilde{\Psi}^{I}$ and $\Psi^{I}$ are therefore just the same. We state this as the following proposition.

Proposition 6.1. Suppose that the bi-*-algebra $\mathscr{G}^{*}$ with unit $e$ and co-product $\Delta$ is a symmetry of a quantum mechanical system with field operators $\Psi_{i}^{I}$ which generate the Hilbert space of physical states from a ground state $|0\rangle$. Then $\Psi_{i}^{I}=\tilde{\Psi}_{i}^{I}$ in the notation (6.13).

Writing $\left(\Psi^{I} \otimes \Psi^{J}\right)_{i j}$ for $\Psi_{i}^{I} \Psi_{j}^{J}$, it follows from the field transformation law that

$$
\Psi_{i}^{I} \Psi_{j}^{J}=\tilde{\Psi}_{i}^{I} \Psi_{j}^{J}=\left(\left(\Psi^{l} \otimes \Psi^{J}\right)\left(\tau^{I} \otimes \tau^{J} \otimes U\right)((\mathrm{id} \otimes \Delta) \Delta(e))\right)_{i j}
$$

This implies

Corollary 6.1. If the symmetry is a weak quasi-triangular quasi Hopf *-algebra then the ordinary product of field operators can be recovered from the covariant product through the same formula (5.12) as for a quasi Hopf algebra but with $\varphi^{-1}$ interpreted as a quasi inverse.

The local braid relations for ordinary products follow then from those for covariant products in the same manner as for a quasi Hopf algebra (see sect. 5). Thus we have the following theorem.

Theorem 6.2. (local braid relations for weak quasi-triangular quasi Hopf symmetries) Suppose that the field operators $\Psi^{I}$ and $\Psi^{J}$ transform covariantly according to representations $\tau^{\prime}$ and $\tau^{J}$ of a weak quasi-triangular quasi Hopf $*$-algebra $\mathscr{G}^{*}$. Suppose that $\mathscr{R}_{k l, i j}^{I J}$ are representation operators of $\mathscr{G}^{*}$ in $\mathscr{H}$ which are defined by eq. (5.13), viz.

$$
\mathscr{R}_{k l, i j}^{I J>}=\left(\tau^{I} \otimes \tau^{J} \otimes U\right)_{k l, i j}\left(\varphi_{213}(R \otimes e) \varphi^{-1}\right)
$$

Then the local braid relations (4.3) are consistent in the sense that both sides of eq. (4.3) transform in the same way under $\mathscr{G}^{*}$. 
Next we discuss consequences of the postulate (6.9). Together with invariance of the ground state (2.12), eq. (6.9) implies that

$$
\left(\Psi^{I} \times \Psi^{J}\right)_{i j}|0\rangle=\Psi_{i}^{I} \Psi_{j}^{J}|0\rangle
$$

and more generally

$$
\left(\Psi^{I_{1}} \times\left(\Psi^{I_{2}} \times \ldots\left(\Psi^{I_{n-1}} \times \Psi^{I_{n}}\right) \ldots\right)\right)_{i_{1} \ldots i_{n}}|0\rangle=\Psi_{i_{1}}^{I_{1}} \Psi_{i_{2}}^{I_{2}} \ldots \Psi_{i_{n-1}}^{I_{n-1}} \Psi_{i_{n}}^{I_{n}}|0\rangle
$$

Stated in words, we have the following proposition.

Proposition 6.2. (fields acting on the ground state) If the weak quasi-triangular quasi Hopf *-algebra is a symmetry of a quantum mechanical system with properties as in definition 3.2 then the ordinary product of field operators agrees with the covariant product, with multiplications performed in the definite order (6.17), when it is applied to the invariant ground state.

In sect. 5 we investigated consequences of Drinfeld's relations (5.25) and (5.26). They remain true when invertibility requirements are weakened so that we have the following theorem.

Theorem 6.3. (local braid relations for composite fields) Suppose that the weak quasi-triangular *-algebra $\mathscr{G}^{*}$ is a symmetry of a quantum mechanical system whose field operators $\Psi^{I}, \Psi^{J}$ satisfy local braid relations (4.3), with matrix $\mathscr{R}$ as described in theorem 6.2. Then the composite fields $\Psi^{I} \times \Psi^{J}$ will also satisfy local braid relations, under appropriate conditions on the arguments of the field operators.

Using quasi-associativity (5.6) of the product $\times$ of fields one can convert to a different order of performing the multiplications. This makes it easy to read off the braid relations for states and for correlation functions. We give an example. Using quasi-associativity (5.6) we have that

$$
\begin{aligned}
\Psi_{j}^{J} \Psi_{k}^{K} \Psi_{l}^{L}|0\rangle & =\left(\Psi^{J} \times\left(\Psi^{K} \times \Psi^{L}\right)\right)_{j k l}|0\rangle \\
& =\left(\left(\Psi^{J} \times \Psi^{K}\right) \times \Psi^{L}\right)_{r p q}|0\rangle\left(\tau^{J} \otimes \tau^{K} \otimes \tau^{L}\right)_{r p q, j k l}\left(\varphi^{-1}\right) \\
& =\left(\Psi^{J} \times \Psi^{K}\right)_{r p} \Psi_{q}^{L}|0\rangle\left(\tau^{J} \otimes \tau^{K} \otimes \tau^{L}\right)_{r p q, j k l}\left(\varphi^{-1}\right)
\end{aligned}
$$

Assuming the arguments of the fields $\Psi^{J}$ and $\Psi^{K}$, which we neglected to write, are in the proper order, we can now apply the local braid relations (5.7) and (5.10) to invert the order of the field operators $\Psi^{J}$ and $\Psi^{K}$. Then one goes backwards, using quasi-associativity and relation (6.9) again. The final result is that

$$
\Psi_{j}^{J} \Psi_{k}^{K} \Psi_{l}^{L}|0\rangle=\Psi_{k^{\prime}}^{K} \Psi_{j^{\prime}}^{J} \Psi_{i^{\prime}}^{L}|0\rangle T_{j^{\prime} k^{\prime} l^{\prime}, j k l}^{J K L}\left(\sigma_{2}\right)
$$


where

$$
T_{j^{\prime} k^{\prime} l^{\prime}, j k l}^{J L}\left(\sigma_{2}\right)=\left(\tau^{J} \otimes \tau^{K} \otimes \tau^{L}\right)_{j^{\prime} k^{\prime} l^{\prime}, j k l}\left(\varphi_{213}(R \otimes e) \varphi^{-1}\right) c_{J K}
$$

where $c_{J K}$ is the phase factor in the braid relations which is left undetermined by the symmetry: it may depend on $J$ and $K$. The notation $T\left(\sigma_{2}\right)$ was chosen to take note of the fact that a representation of the braid group shows up. The braid relation for four point correlation functions is obtained from eq. (6.21) by taking the scalar product with states $\langle 0| \Psi_{i}^{I}$.

Let us finally add a mathematical note. The definition of the covariant product of field operators amounts to introducing a $\mathscr{G}^{*}$-submodule $\mathrm{V}^{I} \times \mathrm{V}^{J}$ of $\mathrm{V}^{I} \otimes \mathrm{V}^{J} \otimes$ $\mathscr{G}^{*}$, and more generally $\mathscr{G}^{*}$-submodules $\left(\ldots\left(V^{I_{1}} \times V^{I_{2}}\right) \ldots \times V^{I_{n}}\right)$, etc. of $V^{I_{1}} \otimes$ $V^{I_{2}} \otimes \ldots \otimes V^{I_{n}} \otimes \mathscr{G}^{*}$. These are the appropriate modules to consider in our context. It may be of interest to note that they are actually bimodules, since right and left action of $\mathscr{G}^{*}$ on them are defined. Bimodules appear naturally [29] when one starts from the algebraic theory of superselection sectors [28].

\section{Covariant adjoints of field operators}

The $R$-element $R=\sum r_{a}^{1} \otimes r_{a}^{2} \in \mathscr{G}^{*} \otimes \mathscr{G}^{*}$ serves another purpose beyond the consistent formulation of local braid relation. It makes it possible to define a covariant adjoint of field operators. This is of interest also in the quantum group context.

A transformation law of adjoints of field operators was stated in proposition 3.1. If there exists and antipodal map $\mathscr{S}$ of the algebra $\mathscr{G}^{*}$ with properties as described in definition 3.1, then the transformation law (3.15) of field operators $\Psi^{l}$ leads to a different transformation law for adjoints $\Psi^{I *}$ of field operators, assuming the $*$-operation on $\mathscr{G}^{*} \otimes \mathscr{G}^{*}$ is defined as in (3.5).

$$
U(\phi) \Psi_{i}^{l *}=\sum_{p} \Psi_{m}^{l *} \tilde{\tau}_{m i}\left(\phi_{p}^{2}\right) U\left(\phi_{p}^{1}\right)
$$

This differs from the transformation law of fields by the interchange of $\phi_{p}^{1}$ and $\phi_{p}^{2}$, apart from the expected appearance of the contragredient representation

$$
\tilde{\tau}(\phi)={ }^{\mathrm{t}} \tau\left(\mathscr{S}^{-1}(\phi)\right)
$$

This complication can be eliminated by defining a covariant adjoint.

Theorem 8 (covariant adjoint of field operators) Suppose that the weak quasitriangular quasi Hopf algebra $\mathscr{G}^{*}$ is a symmetry of the quantum mechanical system with field operators $\Psi_{i}^{l}$ and that the $*$-operation on $\mathscr{G}^{*} \otimes \mathscr{G}^{*}$ is defined 
by eq. (3.5). Make use of $R=\sum r_{a}^{1} \otimes r_{a}^{2}$ to define the "covariant adjoints" $\bar{\Psi}_{i}^{I}$ of field operators $\Psi_{i}^{\prime}$ by

$$
\bar{\Psi}_{i}^{I}=\sum_{a} \Psi_{k}^{I *} \tilde{\tau}_{k i}^{I}\left(r_{a}^{1}\right) U\left(r_{a}^{2}\right)
$$

Then $\bar{\Psi}_{i}^{I}$ transforms covariantly according to the contragredient represetation $\tilde{\tau}^{I}$ of $\tau^{I}$, viz.

$$
U(\phi) \bar{\Psi}_{i}^{I}=\sum_{a} \bar{\Psi}_{k}^{I} \tilde{\tau}_{k i}^{I}\left(\phi_{a}^{1}\right) U\left(\phi_{a}^{2}\right)
$$

This differs from the transformation law for $\Psi^{I}$ only by the appearance of $\tilde{\tau}^{I}$ in place of $\tau^{l}$.

Proof. The main property $\Delta^{\prime}(\phi) R=R \Delta(\phi)$ of the $R$-element reads explicitly

$$
\sum_{a, b} \phi_{b}^{2} r_{a}^{1} \otimes \phi_{b}^{1} r_{a}^{2}=\sum_{a, b} r_{a}^{1} \phi_{b}^{1} \otimes r_{a}^{2} \phi_{b}^{2}
$$

Using this it is straightforward to deduce from the transformation law (7.1) of adjoint field operators that $\bar{\Psi}_{i}^{l}$ transform as stated in the theorem.

Our construction of the covariant adjoint resembles what one does in the theory of the Dirac equation when one introduces an adjoint Dirac spinor $\bar{\Psi}=\Psi^{*} \gamma_{0}$ which transforms covariantly under the Lorentz group.

\section{The comodule picture}

We regarded the Hilbert space $\mathscr{H}$ of physical states as a representation space for $\mathscr{G}^{*}$ because we wanted to generalize the well-known notion of a symmetry in quantum theory where $\mathscr{H}$ is a representation space for a group $\mathscr{G}$ and its group algebra $\mathscr{G}^{*}$. This point of view was proposed by Buchholz et al. [4].

There exists an alternative point of view, introduced by Reshetikhin and Smirnov [15] where the dual $\mathscr{G}$ of $\mathscr{G}^{*}$ plays the central role, and $\mathscr{H}$ is regarded as a co-module for $\mathscr{G}$. In this section we will explain the connection between both pictures.

A representation $U$ of $\mathscr{G}^{*}$ in $\mathscr{H}$ defines a linear map

$$
U(\xi): \mathscr{H} \mapsto \mathscr{H}
$$

for every $\xi \in \mathscr{G}^{*}$. Therefore, $U$ may be regarded as a map

$$
U: \mathscr{G}^{*} \otimes \mathscr{H} \mapsto \mathscr{H}
$$


Given such a representation $U, \mathscr{H}$ becomes a co-module for the dual $\mathscr{G}$ of $\mathscr{G}^{*}$ in a natural way. This means that there is a linear map

$$
\hat{U}: \mathscr{H} \mapsto \mathscr{G} \otimes \mathscr{H}
$$

It is determined by $U$ as follows. Elements $f \in \mathscr{G}$ may be regarded as linear maps of $\mathscr{G}^{*}$ into $\mathbb{C}, \xi \mapsto f(\xi)$. Therefore, elements $f \otimes|v\rangle \in \mathscr{G} \otimes \mathscr{H}$ may be regarded as linear maps $\mathscr{G}^{*} \mapsto \mathscr{H}$, viz.

$$
\xi \mapsto(f \otimes|v\rangle)(\xi)=|v\rangle f(\xi) \in \mathscr{H} .
$$

$\hat{U}$ is defined by

$$
\hat{U}|v\rangle(\xi)=U(\xi)|v\rangle \text { for }|v\rangle \in \mathscr{Z}
$$

If $\mathscr{G}^{*}$ is a bi-algebra then $\mathscr{G}$ is also a bi-algebra. The co-product $\Delta$ in $\mathscr{G}^{*}$ gives the product $\cdot$ in $\mathscr{G}$,

$$
(f \cdot g)(\xi)=f \otimes g(\Delta(\xi))
$$

And the product in $\mathscr{G}^{*}$ yields a co-product $\delta$ in $\mathscr{G}$,

$$
\delta f(\xi, \eta)=f(\xi \eta)
$$

Unit and co-unit are similarly interchanged.

When the co-product in $\mathscr{G}^{*}$ is not co-associative then $\mathscr{G}$ is a nonassociative algebra. So far the co-module picture has been used only for associative $\mathscr{G}$ as far as we know, and we will restrict the discussion to this case to begin with.

We postulated a covariant transformation law of fields under $\mathscr{G}^{*}$. This translates into commutation relations of $\hat{U}$ with fields. In careful notation

$$
\hat{U} \Psi_{i}^{I}=\left(1 \otimes \Psi_{j}^{I}\right)\left(\tau_{j i}^{I} \otimes 1\right) \hat{U}
$$

Herein, the first 1 is the unit element of $\mathscr{G}$ and $1 \otimes \Psi_{i}^{I}$ is a linear map on $\mathscr{G} \otimes \mathscr{H}$ which takes $f \otimes|v\rangle$ into $f \otimes \Psi_{i}^{I}|v\rangle . \tau_{j i}^{\prime}$ are the elements of $\mathscr{G}$ which are defined as linear maps on $\mathscr{G}^{*}, \xi \mapsto \tau_{j i}^{I}(\xi)$ by the representation matrices encountered previously. A product of such elements of $\mathscr{G}$ is defined through eq. (8.6).

Let us demonstrate the validity of commutation relation (8.8). We introduce an orthonormal basis $\left|v_{n}\right\rangle$ in $\mathscr{H}$. We see from eq. (8.5) that

$$
\hat{U}\left|v_{n}\right\rangle=u_{m n} \otimes\left|v_{m}\right\rangle
$$

with $u_{m n} \in \mathscr{G}$ given by

$$
u_{m n}(\xi)=\left\langle v_{m}|U(\xi)| v_{n}\right\rangle
$$


Consider now $\hat{U} \Psi_{i}^{I}\left|v_{n}\right\rangle$. From the definition (8.5) and the covariance property of fields under $\mathscr{G}^{*}$ we deduce

$$
\begin{aligned}
\hat{U} \Psi_{i}^{I}\left|v_{n}\right\rangle(\xi) & =U(\xi) \Psi_{i}^{I}\left|v_{n}\right\rangle=\sum_{a} \Psi_{k}^{I} \tau_{k i}^{I}\left(\xi_{a}^{1}\right) U\left(\xi_{a}^{2}\right)\left|v_{n}\right\rangle \\
& =\sum_{a} \Psi_{k}^{I}\left|v_{m}\right\rangle \tau_{k i}^{I}\left(\xi_{a}^{1}\right) u_{m n}\left(\xi_{a}^{2}\right)
\end{aligned}
$$

if $\Delta(\xi)=\sum \xi_{a}^{1} \otimes \xi_{a}^{2}$. On the other hand

$$
\begin{aligned}
\left(1 \otimes \Psi_{j}^{I}\right)\left(\tau_{j i}^{I} \otimes 1\right) \hat{U}\left|v_{n}\right\rangle(\xi) & =\left(1 \otimes \Psi_{j}^{I}\right)\left(\tau_{j i}^{I} \otimes 1\right)\left(u_{m n} \otimes\left|v_{m}\right\rangle\right)(\xi) \\
& =\Psi_{j}^{I}\left|v_{m}\right\rangle\left(\tau_{j i}^{I} \cdot u_{m n}\right)(\xi) \\
& =\sum_{a} \Psi_{j}^{I}\left|v_{m}\right\rangle \tau_{j i}^{I}\left(\xi_{a}^{I}\right) u_{m n}\left(\xi_{a}^{2}\right)
\end{aligned}
$$

In the last equation, the multiplication law (8.6) in $\mathscr{G}$ was used. We see that both sides of eq. (8.8) are indeed equal when applied to an arbitrary basis vector. This proves the commutation relations $(8.8)$.

The statement of invariance of the ground state translates into

$$
\hat{U}|0\rangle=1 \otimes|0\rangle
$$

where 1 is the unit element in $\mathscr{G}$. Regarded as a linear function on $\mathscr{G}^{*}$ it is given by the co-unit on $\mathscr{G}^{*}$,

$$
\text { 1: } \xi \mapsto \epsilon(\xi)
$$

Let us introduce a shorthand notation. Elements of $\mathscr{G} \otimes \mathscr{H}$ will be written $f|v\rangle$ or $|v\rangle f$ in place of $f \otimes|v\rangle$, and factors $1 \in \mathscr{G}$ will be dropped (but keep eq. (8.17) in mind!) Then, commutation relations with fields and invariance of the ground state reads

$$
\hat{U} \Psi_{i}^{I}=\Psi_{j}^{I} \tau_{j i}^{I} \hat{U}, \quad \hat{U}|0\rangle=|0\rangle
$$

It follows that

$$
\hat{U} \Psi_{i_{1}}^{I_{1}} \ldots \Psi_{i_{n}}^{I_{n}}|0\rangle=\Psi_{j_{1}}^{I_{1}} \ldots \Psi_{j_{n}}^{I_{n}}|0\rangle \tau_{j_{1} i_{1}}^{I_{1}} \cdot \ldots \cdot \tau_{j_{n} i_{n}}^{I_{n}}
$$

When both sides of the state transformation law (8.20) are applied to an arbitrary element $\phi \in \mathscr{G}^{*}$ we recover the old transformation law (3.27) of states.

Elements $\tau \in \mathscr{G}$ commute with operators $\Psi$ on $\mathscr{H}$. However, the multiplication in $\mathscr{G}$ is not commutative if the co-multiplication in $\mathscr{G}^{*}$ is not commutative. Therefore the order of the factors $\tau_{j i}^{I}$ matters. 
From the definition (8.6) we see that the product in $\mathscr{G}$ is in fact equal to the tensor product (of representations) introduced earlier in the sense that

$$
\tau_{i k}^{I} \cdot \tau_{j l}^{J}=\left(\tau^{I} \otimes \tau^{J}\right)_{i j, k l}
$$

The transformation law (8.18) ff. are very much reminiscent of the formula (2.13) for the transformation law of fields under a group, the main difference being that the $\tau_{i k}^{I}$ do not commute. The similarity is still more deceptive when $\tau^{l}$ is the fundamental representation of a matrix group so that $\tau_{i k}^{I}(a)=a_{i k}$. One might then be tempted to write $a_{i k}$ in place of $\tau_{i k}^{I}$ for the function which maps $a$ into $a_{i k}$. But mathematical decency requires that one distinguish between functions and the values which they take. The $\tau_{i k}^{I}$ in eq. (8.20) are generalizations of functions on a group, not values of such functions.

In the case of a quasi Hopf symmetry, the product $\cdot$ in $\mathscr{G}$ is not associative. The formulas obtained so far remain valid, except that it is necessary to put brackets to indicate the order in which multiplications in $\mathscr{G}$ are to be performed. For instance, the state transformation law (8.20) is to be read as

$$
\hat{U} \Psi_{i_{1}}^{I_{1}} \cdots \Psi_{i_{n}}^{I_{n}}|0\rangle=\Psi_{j_{1}}^{I_{1}} \cdots \Psi_{j_{n}}^{I_{n}}|0\rangle\left(\tau_{j_{1} i_{1}}^{I_{1}} \cdot \ldots \cdot\left(\tau_{j_{n-1} I_{n-1}}^{I_{n-1}} \cdot \tau_{j_{n} i_{n}}^{I_{n}}\right) \ldots\right)
$$

\section{The quantum group algebra $\mathrm{U}_{q}\left(\mathrm{sI}_{2}\right)$}

It is instructive to consider as an example the matrix pseudogroup $\mathscr{G}$ whose dual is (or rather: contains) the quantum group algebra $\mathrm{U}_{q}\left(\mathrm{sl}_{2}\right)[8,9] . \mathscr{G}$ is an associative but noncommutative one-parameter deformation * of the algebra Fun $(G)$ of functions on the group $=\operatorname{SL}(2, \mathbb{C})$ of complex unimodular $2 \times 2$ matrices $a=\left(a_{i j}\right)_{i, j=1,2}$.

Let us first consider the commutative algebra Fun(SL $(2, \mathbb{C}))$. This algebra is generated by simple functions $\tau_{i j}, i, j=1,2$. They are defined by

$$
\tau_{i j}(a)=a_{i j}
$$

In the old language, $\tau$ is the fundamental representation. These generators of $\mathscr{G}$ obey the relations

$$
\begin{aligned}
& \operatorname{det} \tau \equiv \tau_{11} \cdot \tau_{22}-\tau_{12} \cdot \tau_{21}=1, \\
& \tau_{i j} \cdot \tau_{k l}=\tau_{k l} \cdot \tau_{i j} .
\end{aligned}
$$

* GL(2) possesses a two-parameter deformation [31]. 
We remember that the multiplication - of functions is pointwise defined, $f \cdot g(a)=$ $f(a) g(a)$. Eq. (9.3) expresses commutativity of this product.

The deformations $\mathscr{G}$ are parametrized by a complex number $q$. $\mathscr{G}$ is again generated by elements $\tau_{i j}$, but the relations (9.2) and (9.3) get replaced by

$$
\begin{aligned}
\operatorname{det} \tau & \equiv \tau_{11} \cdot \tau_{22}-q^{1 / 2} \tau_{12} \cdot \tau_{21}=1, \\
\mathscr{R}_{i k, r s} \tau_{r l} \cdot \tau_{s w} & =\tau_{k l} \cdot \tau_{i m} \mathscr{R}_{m l, l w},
\end{aligned}
$$

where $\mathscr{R}$ is our old element $R \in \mathscr{G}^{*} \otimes \mathscr{G}^{*}$ in the fundamental representation $\tau$ of $\mathscr{G}^{*}$

$$
\mathscr{R}_{i k, r s}=(\tau \otimes \tau)_{i k, r s}(R)
$$

When rows and columns are labelled in lexicographic order, $\mathscr{R}$ has the explicit form

$$
\mathscr{R}=\left(\begin{array}{cccc}
q^{1 / 2} & 0 & 0 & 0 \\
0 & 1 & 0 & 0 \\
0 & q^{1 / 2}-q^{-1 / 2} & 1 & 0 \\
0 & 0 & 0 & q^{1 / 2}
\end{array}\right)
$$

In the alternative notation

$$
\tau=\left(\begin{array}{ll}
\tau_{11} & \tau_{12} \\
\tau_{21} & \tau_{22}
\end{array}\right)=\left(\begin{array}{ll}
a & b \\
c & d
\end{array}\right)
$$

the relations (9.5) are given by

$$
\begin{aligned}
& a b=q^{1 / 2} b a, \quad a c=q^{1 / 2} c a, \quad a d-d a=\left(q^{1 / 2}-q^{-1 / 2}\right) b c, \\
& c d=q^{1 / 2} d c, \quad b d=q^{1 / 2} d b, \quad b c=c b .
\end{aligned}
$$

It remains to exhibit the generators of $\mathscr{G}$ as complex linear functions on $\mathrm{U}_{q}\left(\mathrm{sl}_{2}\right)$. The formulas that do this have been found by Reshetikhin et al. [23].

The quantum group algebra $\mathrm{U}_{q}\left(\mathrm{sl}_{2}\right)$ is generated by elements $q^{ \pm H / 2}$ and $S_{ \pm}$ subject to the relations

$$
\begin{gathered}
q^{H / 2} q^{-H / 2}=q^{-H / 2} q^{H / 2}=1, \\
q^{H / 2} S_{ \pm}=q^{ \pm 1 / 2} S_{ \pm} q^{H / 2} \\
{\left[S_{+}, S_{-}\right]=\frac{q^{H}-q^{-H}}{q^{1 / 2}-q^{-1 / 2}}}
\end{gathered}
$$


Here and in the following we write 1 in place of $e$ for the unit element in $\mathscr{G}^{*}=\mathrm{U}_{q}\left(\mathrm{sl}_{2}\right)$.

The co-multiplication is given by

$$
\begin{aligned}
\Delta\left(q^{ \pm H / 2}\right) & =q^{ \pm H / 2} \otimes q^{ \pm H / 2}, \\
\Delta\left(S_{ \pm}\right) & =S_{ \pm} \otimes q^{H / 2}+q^{-H / 2} \otimes S_{ \pm}
\end{aligned}
$$

If $|q|=1$, a $*$-operation can be defined by

$$
S_{ \pm}^{*}=S_{\mp}, \quad\left(q^{ \pm H / 2}\right)^{*}=q^{\mp H / 2}
$$

and this is an automorphism satisfying $\Delta\left(S^{*}\right)=\Delta(S)^{*}$ provided we adopt convention (3.5) for the adjoint on $\mathscr{G}^{*} \otimes \mathscr{G}^{*}$.

The co-unit is

$$
\epsilon\left(q^{ \pm H / 2}\right)=1, \quad \epsilon\left(S_{ \pm}\right)=0
$$

and the antipodal map $\mathscr{S}$ acts as

$$
q^{\mp H / 2}=\mathscr{S}\left(q^{ \pm H / 2}\right), \quad-q^{ \pm 1 / 2} \mathrm{~S}_{ \pm}=\mathscr{P}\left(\mathrm{S}_{ \pm}\right)
$$

It is convenient to use matrix notation again. Following Reshetikhin et al. [23] one puts

$$
\begin{array}{cl}
L_{+}=\left(\begin{array}{cc}
q^{H / 2} & \left(q^{1 / 2}-q^{-1 / 2}\right) S_{+} \\
0 & q^{-H / 2}
\end{array}\right), & L_{-}=\left(\begin{array}{cc}
q^{-H / 2} & 0 \\
\left(q^{-1 / 2}-q^{1 / 2}\right) S_{-} & q^{H / 2}
\end{array}\right), \\
L_{ \pm}^{1}=L_{ \pm} \otimes 1, & L_{ \pm}^{2}=1 \otimes L_{ \pm}, \\
T^{1}=\tau \otimes 1, & T^{2}=1 \otimes \tau \\
R^{+}=P R P, & R^{-}=R^{-1}
\end{array}
$$

with $P(a \otimes b)=b \otimes a$.

In this notation the action of generators $q^{ \pm H / 2}, S_{ \pm}$of $\mathrm{U}_{q}\left(\mathrm{sl}_{2}\right)$ on generators $\tau_{i j}$ of $\mathscr{G}$ is given by

$$
L_{ \pm}^{1}: T^{2} \mapsto R^{ \pm}
$$




\section{Weak quasi Hopf algebras associated with $U_{q}\left(s l_{2}\right)$ for $q^{p}=1$}

Now we turn to the problem of how to truncate the algebra $\mathrm{U}_{q}\left(\mathrm{sl}_{2}\right)$ in such a way that its unphysical representations are eliminated but constructions of physical interest, such as the construction of tensor products of physical representations, remain possible.

The quantum group algebra $\mathrm{U}_{q}\left(\mathrm{sI}_{2}\right)$ with $q^{p}=1$ has physical representations $\pi^{K}, 2 K=0,1, \ldots, p-2$, which act in representation spaces $V^{K}$. The tensor product of two such physical representations decomposes in general into physical representations, plus unphysical subrepresentations with quantum dimension 0 .

Let $\mathscr{J}$ be the ideal in $\mathrm{U}_{q}\left(\mathrm{sl}_{2}\right)$ which is annihilated by all physical $\pi^{K}$. We set

$$
\mathscr{G}^{*}=\mathrm{U}_{q}\left(\mathrm{sl}_{2}\right) / \mathscr{J}
$$

Equivalently,

$$
\mathscr{G}^{*}=\pi\left(\mathrm{U}_{q}\left(\mathrm{sl}_{2}\right)\right), \quad \pi=\underset{K \text { phys }}{\bigoplus} \pi^{K}
$$

Let $\otimes_{q}$ be the tensor product of $\mathrm{U}_{q}\left(\mathrm{sl}_{2}\right)$ representations. It comes from a co-product $\Delta_{q}$ for $\mathrm{U}_{q}\left(\mathrm{sl}_{2}\right)$. In application to conformal field theory one invents an extra rule which says that the extra terms represented by dots in the tensor product decomposition

$$
\begin{aligned}
& \pi^{I} \frac{\otimes}{q} \pi^{J}=\underset{|I-J| \leqslant K \leqslant u(I J)}{\oplus} \pi^{K}+\cdots, \\
& u(I J) \equiv \min \{|I+J|, p-2-I-J\}
\end{aligned}
$$

are to be thrown away. The left out part has quantum dimension 0 [16]. Supplemented with this extra rule, the tensor product decomposition reproduces the fusion rules of minimal conformal models.

It is customary to define a truncated tensor product $\otimes$ of physical representations of $\mathrm{U}_{q}\left(\mathrm{sl}_{2}\right)$ such that the unwanted terms in the tensor product decomposition (10.2) are absent,

$$
\pi^{I} \otimes \pi^{J}=\bigoplus_{|I-J| \leqslant K \leqslant u(I J)} \pi^{K}
$$

The explicit construction uses a projection operator which is furnished by the Clebsch-Gordan maps for $\mathrm{U}_{q}\left(\mathrm{sl}_{2}\right)$,

$$
C_{q}(I J \mid K): \quad \mathrm{V}^{I} \otimes \mathrm{V}^{J} \mapsto \mathrm{V}^{K}
$$


By definition, they enjoy the intertwining property

$$
C_{q}(I J \mid K)\left(\pi^{I} \frac{\otimes}{q} \pi^{J}\right)(\xi)=\pi^{K}(\xi) C_{q}(I J \mid K)
$$

for all $\xi$. The nonassociative truncated tensor product $\otimes$ of physical representations of $\mathrm{U}_{q}\left(\mathrm{sl}_{2}\right)$ is defined by

$$
\begin{aligned}
\left(\pi^{I} \otimes \pi^{J}\right)(\xi) & =\sum_{|I-J| \leqslant K \leqslant u(J)} C_{q}(I J \mid K)^{*} \pi^{K}(\xi) C_{q}(I J \mid K) \\
& =\left(\pi^{I} \otimes \pi^{J}\right)\left(\Delta_{q}(\xi)\right) P_{I J},
\end{aligned}
$$

with

$$
P_{I J}=\sum_{|I-J| \leqslant K \leqslant u(J)} C_{q}(I J \mid K)^{*} C_{q}(I J \mid K) .
$$

This tensor product is at the same time a representation of $\mathscr{G}^{*}$, i.e. it annihilates I.

We may now state the main result of this section in the following theorem.

Theorem 10.1. (truncated quantum groups) Let $q^{p}=1$. There exists a weak quasi-triangular quasi Hopf $*$-algebra $\mathscr{G}^{*}$ such that the indecomposable representations of $\mathscr{G}^{*}$ are precisely the physical representations of $\mathrm{U}_{q}\left(\mathrm{sl}_{2}\right)$, and the tensor product $\otimes$ of representations of $\mathscr{G}^{*}$, which is furnished by its co-product $\Delta$, agrees with the truncated tensor product of physical representations of $\mathrm{U}_{q}\left(\mathrm{sl}_{2}\right)$.

As an algebra, $\mathscr{G}^{*}=\mathrm{U}_{q}\left(\mathrm{sl}_{2}\right) / \mathscr{J}$, where $\mathscr{J}$ is the ideal in $\mathrm{U}_{q}\left(\mathrm{sl}_{2}\right)$ which is annhilated by all its physical representations. The co-product $\Delta$ and $R$ are obtained from the co-product $\Delta_{\mathrm{q}}$ and $R$-element $R_{q}$ of $\mathrm{U}_{q}\left(\mathrm{sl}_{2}\right)$ as follows:

$$
\Delta(\xi)=P \Delta_{q}(\xi), \quad R=R_{q} P
$$

where $P \in \mathscr{G}^{*} \otimes \mathscr{G}^{*}$ is a projector whose representative in $\mathrm{U}_{\mathrm{q}}\left(\mathrm{sl}_{2}\right) \otimes \mathrm{U}_{\mathrm{q}}\left(\mathrm{sl}_{2}\right)$ is given by eq. (10.15), with projectors $P^{K} \in \mathrm{U}_{q}\left(\mathrm{sl}_{2}\right)$ obtained from eqs. (10.11) (10.14). The associator $\varphi \in \mathscr{G}^{*} \otimes \mathscr{G}^{*} \otimes \mathscr{G}^{*}$ exists and is determined by its action on basis vectors in triple truncated tensor products of dual representation spaces, eq. (10.28), with $6 j$-symbols (10.34) as coefficients.

The proof will proceed in several stages. As a *-algebra with unit $e, \mathscr{G}^{*}$ is defined by eq. (10.1) (and the *-operation on $\mathscr{G}^{*} \otimes \mathscr{G}^{*}$ is given by eq. (3.5)). This ensures that the indecomposable representations of $\mathscr{G}^{*}$ are precisely the physical representations of $\mathrm{U}_{q}\left(\mathrm{sI}_{2}\right)$. It remains to exhibit the co-product, co-unit, antipode, $R$, and $\varphi$ with the appropriate properties. Co-unit and antipode will be those inherited from $\mathrm{U}_{q}\left(\mathrm{sl}_{2}\right)$. We turn to the construction of the co-product $\Delta$. 
Suppose for a moment that there exists an element $P$ of $\mathrm{U}_{q}\left(\mathrm{sI}_{2}\right) \otimes \mathrm{U}_{q}\left(\mathrm{sI}_{2}\right)$ such that

$$
P_{I J}=\left(\pi^{I} \otimes \pi^{J}\right)(P)
$$

Then we may define a co-product $\Delta$ in $\mathscr{G}^{*}$ such that

$$
\left(\pi^{I} \otimes \pi^{J}\right)(\xi)=\left(\pi^{I} \otimes \pi^{J}\right)(\Delta(\xi))
$$

This is achieved by setting

$$
\Delta(\xi)=P \Delta_{q}(\xi)
$$

Property $(10.10)$ is immediate. We show that $\Delta(\xi)$ is well defined for $\xi \in \mathscr{G}^{*}$, i.e. depends on $X \in \mathrm{U}_{q}\left(\mathrm{sl}_{2}\right)$ only through its equivalence class $\xi \bmod \mathscr{J}$ in $\mathscr{G}^{*}$. In view of the definition (10.1) it suffices to show that for arbitrary physical $I, J$ we have $\left(\pi^{I} \otimes \pi^{J}\right)(\Delta(Z))=0$ if $Z \in \mathscr{J}$. But this follows right away from property (10.10) and eq. (10.6).

We will construct the desired projector from the Casimir invariant $\mathscr{C} \in \mathrm{U}_{q}\left(\mathrm{sl}_{2}\right)$,

$$
\mathscr{C}=S^{-} S^{+}+\left(\frac{q^{\left(H+\frac{1}{2}\right) / 2}-q^{-\left(H+\frac{1}{2}\right) / 2}}{q^{1 / 2}-q^{-1 / 2}}\right)^{2}-\left(\frac{q^{1 / 4}-q^{-1 / 4}}{q^{1 / 2}-q^{-1 / 2}}\right)^{2} .
$$

It has eigenvalues

$$
\lambda_{J}=\left[J+\frac{1}{2}\right]_{q}^{2}-\left[\frac{1}{2}\right]_{q}^{2}, \quad J=0, \frac{1}{2}, 1, \ldots
$$

where we use the standard notation $[x]_{q}=\left(q^{x / 2}-q^{-x / 2}\right)\left(q^{1 / 2}-q^{-1 / 2}\right)^{-1}$. The Casimir does not suffice to make a global distinction between physical representations and unphysical ones because of the symmetry of $\lambda_{K}$ under $K \mapsto p-1-K$. But fortunately this is not needed, either. Given $I, J$ we say that $K$ is "permissible" if $|I-J| \leqslant K \leqslant|I+J|$ and $K \leqslant u(I, J)$ and "unwanted" if $|I-J| \leqslant K \leqslant$ $|I+J|$ but $K>u(I, J)$. We see that it can never happen that $K$ is unwanted but its brother $p-1-K$ is permissible, or the other way round.

Let $\theta_{K}$ be the real function, defined on the set $\mathrm{E}$ of distinct possible eigenvalues of $\mathscr{E}$, which is equal to 1 for argument $x=\lambda_{K}$ and 0 otherwise,

$$
\theta_{K}(x)=\prod_{r \in E, r \neq \lambda_{K}}(x-r)\left(\lambda_{K}-r\right)^{-1} .
$$

We consider the projectors

$$
P^{K}=\theta_{K}(\mathscr{E}) \in \mathrm{U}_{q}\left(\mathrm{sl}_{2}\right)
$$


and set

$$
P=\sum_{I, J \text { phys }}\left(P^{I} \otimes P^{J}\right) \sum_{|I-J| \leqslant K \leqslant u(I J)} \Delta_{q}\left(P^{K}\right) \in \mathrm{U}_{q}\left(\mathrm{sl}_{2}\right) \otimes \mathrm{U}_{q}\left(\mathrm{sl}_{2}\right) .
$$

$P^{I}$ commutes with all elements of $U_{q}\left(\mathrm{sI}_{2}\right)$. Therefore $P$ is a sum of products of commuting projectors. It has the desired properties because of the intertwining property of Clebsch-Gordan maps, and because there is no need to discriminate between representations which $P^{K}$ fails to distinguish on account of the symmetry $K \mapsto p-1-K$.

The one-dimensional representation $\pi^{0}$ gives the co-unit,

$$
\epsilon(\xi)=\pi^{0}(\xi)
$$

The co-unit will therefore remain the same as in $\mathrm{U}_{q}\left(\mathrm{sl}_{2}\right)$. When $I=0$ or $J=0$ then the restricted tensor product (10.6) agrees with the old tensor product $\pi^{I} \otimes q_{q} \pi^{J}$. Therefore

$$
\pi^{K}(\xi)=\left(\pi^{K} \otimes \pi^{0}\right)(\xi)=\left(\pi^{K} \otimes \pi^{0}\right)(\Delta(\xi))=\pi^{K}((\mathrm{id} \otimes \epsilon) \Delta(\xi)),
$$

and similarly for $\pi^{0} \otimes \pi^{K}$. This is true for all physical representations $\pi^{K}$. Recalling definition (10.1), it follows that the relation

$$
(\mathrm{id} \otimes \epsilon) \Delta=\mathrm{id}=(\epsilon \otimes \mathrm{id}) \Delta
$$

holds true.

This completes the construction of the co-product $\Delta$ for $\mathscr{G}^{*}$.

An $R$-element and its pseudo-inverse exist such that $R \Delta(\xi)=\Delta^{\prime}(\xi) R$ and relations (6.7) and (6.8) hold. In terms of the $R$-element $R_{q}$ of $\mathrm{U}_{q}\left(\mathrm{sl}_{2}\right)$ it is given by

$$
\begin{gathered}
R=R_{q} \Delta(e)=\Delta^{\prime}(e) R_{q}, \\
R^{-1}=R_{q}^{-1} \Delta^{\prime}(e)=\Delta(e) R_{q}^{-1} .
\end{gathered}
$$

Next we will show that there exists an element $\varphi \in \mathscr{G}^{*} \otimes \mathscr{G}^{*} \otimes \mathscr{G}^{*}$ which possesses a quasi-inverse and which makes the co-product quasi-coassociative in the sense that eq. (5.1) holds, viz.

$$
\varphi(\Delta \otimes \mathrm{id}) \Delta(\xi)=(\mathrm{id} \otimes \Delta) \Delta(\xi) \varphi \text { for all } \xi \in \mathscr{G}^{*} .
$$

One of the purposes of the associator $\varphi$ is to ensure that the restricted tensor products of representations $\left(\pi^{I} \otimes \pi^{J}\right) \otimes \pi^{K}$ and $\pi^{I} \underline{\otimes}\left(\pi^{J} \otimes \pi^{K}\right)$ are equivalent. By definition,

$$
\begin{aligned}
& \left(\left(\pi^{I} \otimes \pi^{J}\right) \otimes \pi^{K}\right)(\xi)=\pi^{I} \otimes \pi^{J} \otimes \pi^{K}((\Delta \otimes \mathrm{id}) \Delta(\xi)), \\
& \left(\pi^{I} \otimes\left(\pi^{J} \otimes \pi^{K}\right)\right)(\xi)=\pi^{I} \otimes \pi^{J} \otimes \pi^{K}((\mathrm{id} \otimes \Delta) \Delta(\xi)) .
\end{aligned}
$$


Both are representations of $\mathrm{U}_{q}\left(\mathrm{sl}_{2}\right)$ which are at the same time representations of $\mathcal{G}^{*}$. It is well known that equivalence of these two representations is indeed true $[19,32]$. There will therefore be maps

$$
\varphi_{I J K}: \quad \mathrm{V}^{I} \otimes \mathrm{V}^{J} \otimes \mathrm{V}^{K} \mapsto \mathrm{V}^{I} \otimes \mathrm{V}^{J} \otimes \mathrm{V}^{K}
$$

such that

$$
\varphi_{I J K}\left(\left(\pi^{I} \otimes \pi^{J}\right) \otimes \pi^{K}\right)(\xi)=\left(\pi^{I} \underline{\otimes}\left(\pi^{J} \otimes \pi^{K}\right)\right)(\xi) \varphi_{I J K}
$$

for all $\xi \in \mathscr{G}^{*}$, and $\varphi_{I J K}$ possesses a quasi-inverse $\varphi_{I J K}^{-1}$ such that

$$
\begin{aligned}
& \varphi_{I J K} \varphi_{I J K}^{-1}=\left(\pi^{I} \underline{\otimes}\left(\pi^{J} \otimes \pi^{K}\right)\right)(e)=\left(\pi^{I} \otimes \pi^{J} \otimes \pi^{K}\right)((\mathrm{id} \otimes \Delta) \Delta(e)), \\
& \left.\varphi_{I J k}^{-1} \varphi_{I J K}=\left(\left(\pi^{I} \otimes \pi^{J}\right) \otimes \pi^{K}\right)(e)=\left(\pi^{I} \otimes \pi^{J} \otimes \pi^{K}\right)(\Delta \otimes \mathrm{id}) \Delta(e)\right) .
\end{aligned}
$$

We seek $\varphi \in \mathscr{G}^{*} \otimes \mathscr{G}^{*} \otimes \mathscr{G}^{*}$, or a representative of it in $\mathrm{U}_{q}\left(\mathrm{sl}_{2}\right) \otimes \mathrm{U}_{q}\left(\mathrm{sl}_{2}\right) \otimes \mathrm{U}_{q}\left(\mathrm{sl}_{2}\right)$, such that

$$
\varphi_{I J K}=\left(\pi^{I} \otimes \pi^{J} \otimes \pi^{K}\right)(\varphi)
$$

Let $\mathscr{H}^{I}$ be the full matrix algebra which consists of $(2 I+1) \times(2 I+1)$ matrices. By construction, $\mathscr{G}^{*}$ is a finite-dimensional semisimple algebra. Therefore it is a direct sum of full matrix algebras. Since the representations $\pi^{K}$ are irreducible, it follows from the definition (10.1) that $\pi^{K}\left(\mathscr{G}^{*}\right)=\mathscr{M}^{K}$. To every matrix $m \in \mathscr{M}^{K}$ there will therefore exist some element $\mu \in \mathscr{G}^{*}$ such that $m=\pi^{K}(\mu)$. By definition

$$
\varphi_{I J K} \in \mathscr{M}^{I} \otimes \mathscr{M}^{J} \otimes \mathscr{M}^{K}
$$

It follows that there exists $\phi_{I J K} \in \mathscr{M}^{I} \otimes \mathscr{M}^{J} \otimes \mathscr{G}^{*}$ such that

$$
\varphi_{I J K}=\left(\mathrm{id} \otimes \mathrm{id} \otimes \pi^{K}\right)\left(\phi_{I J K}\right) .
$$

Setting

$$
\phi_{I J}=\sum_{K} P^{K} \phi_{I J K}
$$

we have that

$$
\pi^{K}\left(\phi_{I J}\right)=\varphi_{I J K}
$$

for all $K . \phi_{I J}$ is a sum of terms $m^{I} \otimes m^{J} \otimes \xi_{I J}$ with matrices $m^{I}, m^{J}$ and $\xi_{I J} \in \mathscr{G}^{*}$. Next we may use the same argument to write $m^{J}=\pi^{J}\left(\phi_{I J}^{\prime}\right)$ and define

$$
\phi_{I}^{\prime}=\sum_{J} P^{J} \phi_{I J}^{\prime} \otimes \xi_{I J} \in \mathscr{G}^{*} \otimes \mathscr{G}^{*}
$$


And so it goes. Repeating the same argument once more we arrive finally at an element $\varphi \in \mathscr{G}^{*} \otimes \mathscr{G}^{*} \otimes \mathscr{G}^{*}$ with the desired property (10.25). This completes the construction of $\varphi$. The existence of its quasi-inverse $\varphi^{-1}$ follows in the same way.

Given $\varphi_{I J K}, \varphi$ is unique. We will now show that there exists an element $\varphi \in \mathscr{G}^{*} \otimes \mathscr{G}^{*} \otimes \mathscr{G}^{*}$, so that Drinfeld's relations (5.1), (5.5), (5.25) and (5.26) hold. To this effect we will give an explicit definition of $\varphi_{I J K}$ by specifying its action on basis vectors. We will no longer distinguish in notation between $\varphi$ and $\varphi_{I J K}$ in the following. More generally we write $\zeta$ in place of $\left(\pi^{l} \otimes \pi^{l} \otimes \pi^{K}\right)(\zeta)$, etc.

$\mathscr{G}^{*}$ acts on the duals $\hat{\mathrm{V}}^{I}$ of representation spaces $\mathrm{V}^{\prime}$ from the right, $\hat{e}_{i}^{\prime} \xi=$ $\tau_{i i^{\prime}}^{l}(\xi) \hat{e}_{i^{\prime}}^{l}$ for $\xi \in \mathscr{G}^{*}$. A truncated tensor product $\otimes$ is not only defined for representations, but also for basis vectors $\hat{e}_{i}^{l}$ in the spaces $\hat{\mathrm{V}}^{I}$,

$$
\hat{e}_{i}^{I} \otimes \hat{e}_{j}^{J}=\hat{e}_{i}^{I} \otimes \hat{e}_{j}^{J} P_{I J}=\hat{e}_{i}^{I} \otimes \hat{e}_{j}^{J} \Delta(e)
$$

The definition extends to reducible representations, so that multiple products are also defined. In particular

$$
\begin{aligned}
& \hat{e}_{i}^{I} \otimes\left(\hat{e}_{j}^{J} \otimes \hat{e}_{k}^{K}\right)=\hat{e}_{i}^{I} \otimes \hat{e}_{j}^{J} \otimes \hat{e}_{k}^{K}(\mathrm{id} \otimes \Delta) \Delta(e), \\
& \left(\hat{e}_{i}^{I} \otimes \hat{e}_{j}^{J}\right) \otimes \hat{e}_{k}^{K}=\hat{e}_{i}^{I} \otimes \hat{e}_{i}^{J} \otimes \hat{e}_{k}^{K}(\Delta \underline{\otimes \mathrm{id}}) \Delta(e) .
\end{aligned}
$$

Vectors $\hat{e}_{i}^{I} \otimes\left(\hat{e}_{j}^{J} \otimes \hat{e}_{k}^{K}\right)$ span a subspace $\hat{\mathrm{V}}_{i}^{I} \otimes\left(\hat{\mathrm{V}}_{j}^{J} \otimes \hat{\mathrm{V}}_{k}^{K}\right)$ of $\hat{\mathrm{V}}_{i}^{I} \otimes \hat{\mathrm{V}}_{j}^{J} \otimes \hat{\mathrm{V}}_{k}^{K}$, and vectors $\left(\hat{e}_{i}^{I} \otimes \hat{e}_{j}^{J}\right) \otimes \hat{e}_{k}^{K}$ span another subspace $\left(\hat{\mathrm{V}}_{i}^{I} \otimes \hat{\mathrm{V}}_{j}^{J}\right) \otimes \hat{\mathrm{V}}_{k}^{K}$. The vectors $\left(\hat{e}_{i}^{I} \otimes \hat{e}_{j}^{J}\right) \otimes \hat{e}_{k}^{K}$ will sometimes be called "physical states". They are not all linearly independent, but $\hat{V}_{i}^{I} \otimes\left(\hat{\mathrm{V}}_{j}^{J} \otimes \hat{\mathrm{V}}_{k}^{K}\right)$ is spanned by the linearly independent vectors

$$
\sum_{i j k p} C\left[\begin{array}{ccc}
l & P & L \\
i & p & l
\end{array}\right]_{q} C\left[\begin{array}{lll}
J & K & P \\
j & k & p
\end{array}\right]_{q} \hat{e}_{i}^{I} \otimes \hat{e}_{j}^{J} \otimes \hat{e}_{k}^{K}
$$

with $|K-J| \leqslant P \leqslant u(K J)$ and $|P-I| \leqslant L \leqslant u(P I)$. The symbols $\left.C_{i j k}^{I J K}\right]_{q}$ denote the usual Clebsch-Gordan coefficients for $\mathrm{U}_{q}\left(\mathrm{sl}_{2}\right)$ which are determined by the above mentioned Clebsch-Gordan maps. They will be the Clebsch-Gordan coefficients for $\mathscr{G}^{*}$ at the same time when $|I-J| \leqslant K \leqslant u(I J)$. The other ClebschGordan coefficients for $\mathscr{G}^{*}$ vanish.

We make an ansatz for the action of $\varphi$ on these basis vectors,

$$
\begin{gathered}
\sum_{i j k p} C\left[\begin{array}{lll}
I & P & L \\
i & p & l
\end{array}\right]_{q} C\left[\begin{array}{lll}
J & K & P \\
j & k & p
\end{array}\right]_{q} \hat{e}_{i}^{I} \otimes \hat{e}_{j}^{J} \otimes \hat{e}_{k}^{K} \varphi \\
=\sum_{Q, i j k q} F_{P Q}\left[\begin{array}{ll}
J & I \\
K & L
\end{array}\right]_{C}\left[\begin{array}{lll}
I & J & Q \\
i & j & q
\end{array}\right]_{q} C\left[\begin{array}{lll}
Q & K & L \\
q & k & l
\end{array}\right]_{q} \hat{e}_{i}^{I} \otimes \hat{e}_{j}^{J} \otimes \hat{e}_{k}^{K} .
\end{gathered}
$$


The action of $\varphi$ is extended to all of $\hat{\mathrm{V}}^{I} \otimes \hat{\mathrm{V}}^{J} \otimes \hat{\mathrm{V}}^{K}$ by imposing the requirement that $\varphi=($ id $\otimes \Delta) \Delta(e) \varphi$. By this ansatz, the intertwining property (5.1) of $\varphi$ is assured for arbitrary choice of the complex coefficients $F_{P Q}\left[L_{K}^{J} L_{L}^{\prime}\right]$.

This is verified by a short calculation as follows. Since

$(\mathrm{id} \otimes \Delta) \Delta(\xi) \varphi=(\mathrm{id} \otimes \Delta) \Delta(\xi)(\mathrm{id} \otimes \Delta) \Delta(e) \varphi=(\mathrm{id} \otimes \Delta) \Delta(e)(\mathrm{id} \otimes \Delta) \Delta(\xi) \varphi$

it suffices to consider (id $\otimes \Delta) \Delta(\xi)_{\varphi}$ on the physical states in the tensor product decomposition. Using the following transformation laws

$$
\begin{gathered}
\sum_{i j k q} C\left[\begin{array}{lll}
I & J & Q \\
i & j & q
\end{array}\right]_{q} C\left[\begin{array}{ccc}
Q & K & L \\
q & k & l
\end{array}\right]_{q} \hat{e}_{i}^{I} \otimes \hat{e}_{j}^{J} \otimes \hat{e}_{k}^{K}(\Delta \otimes \mathrm{id}) \Delta(\xi) \\
=\sum_{i j k q l^{\prime}} C\left[\begin{array}{lll}
I & J & Q \\
i & j & q
\end{array}\right]_{q} C\left[\begin{array}{ccc}
Q & K & L \\
q & k & l^{\prime}
\end{array}\right]_{q} \tau_{l l^{\prime}}^{\prime}(\xi) \hat{e}_{i}^{I} \otimes \hat{e}_{j}^{J} \otimes \hat{e}_{k}^{K}, \\
\sum_{i j k p} C\left[\begin{array}{lll}
J & K & P \\
j & k & p
\end{array}\right]_{q} C\left[\begin{array}{lll}
I & P & L \\
i & p & l
\end{array}\right]_{q} \hat{e}_{i}^{I} \otimes \hat{e}_{j}^{J} \otimes \hat{e}_{k}^{K}(\mathrm{id} \otimes \Delta) \Delta(\xi) \\
=\sum_{i j k p l^{\prime}} C\left[\begin{array}{lll}
J & K & P \\
j & k & p
\end{array}\right]_{q} C\left[\begin{array}{lll}
I & P & L \\
i & p & l^{\prime}
\end{array}\right]_{q} \tau_{l l^{\prime}}^{L}(\xi) \hat{e}_{i}^{I} \otimes \hat{e}_{j}^{J} \otimes \hat{e}_{k}^{K},
\end{gathered}
$$

we calculate

$$
\begin{aligned}
& \sum_{i j k p} C\left[\begin{array}{ccc}
I & P & L \\
i & p & l
\end{array}\right]_{q} C\left[\begin{array}{ccc}
J & K & P \\
j & k & p
\end{array}\right]_{q} \hat{e}_{i}^{I} \otimes \hat{e}_{j}^{J} \otimes \hat{e}_{k}^{K}(\mathrm{id} \otimes \Delta) \Delta(\xi) \varphi \\
& =\sum_{i j k p l^{\prime}} C\left[\begin{array}{ccc}
I & P & L \\
i & p & l^{\prime}
\end{array}\right]_{q} C\left[\begin{array}{ccc}
J & K & P \\
j & k & p
\end{array}\right]_{q} \tau_{l l^{\prime}}^{L}(\xi) \hat{e}_{i}^{I} \otimes \hat{e}_{j}^{J} \otimes \hat{e}_{k}^{K} \varphi \\
& =\sum_{Q, i j k q l^{\prime}} F_{P Q}\left[\begin{array}{ll}
J & I \\
K & L
\end{array}\right] C\left[\begin{array}{ccc}
I & J & Q \\
i & j & q
\end{array}\right]_{q} C\left[\begin{array}{ccc}
Q & K & L \\
q & k & l^{\prime}
\end{array}\right]_{q} \tau_{l l^{\prime}}^{\prime}(\xi) \hat{e}_{i}^{\prime} \otimes \hat{e}_{j}^{J} \otimes \hat{e}_{k}^{K} \\
& =\sum_{Q, i j k q} F_{P Q}\left[\begin{array}{ll}
J & I \\
K & L
\end{array}\right]\left[\begin{array}{ccc}
I & J & Q \\
i & j & q
\end{array}\right]_{q} C\left[\begin{array}{ccc}
Q & K & L \\
q & k & l^{\prime}
\end{array}\right]_{q} \\
& \times \tau_{l l}^{L} \cdot(\xi) \hat{e}_{i}^{I} \otimes \hat{e}_{j}^{J} \otimes \hat{e}_{k}^{K}(\Delta \otimes \mathrm{id}) \Delta(\xi) \\
& =\sum_{i j k p} C\left[\begin{array}{lll}
I & P & L \\
i & p & l
\end{array}\right]_{q} C\left[\begin{array}{ccc}
J & K & P \\
j & k & p
\end{array}\right]_{q} \hat{e}_{i}^{I} \otimes \hat{e}_{j}^{J} \otimes \hat{e}_{k}^{K} \varphi(\Delta \otimes \mathrm{id}) \Delta(\xi) .
\end{aligned}
$$


This completes the demonstration of the intertwining property (5.1). Now we turn to the other properties of $\varphi$ in a weak quasi Hopf algebra.

Eqs. (5.25) and (5.26) impose further restrictions on the coefficients $F_{P Q}[\because]$. Acting with (id $\otimes \mathrm{id} \otimes \Delta)(\varphi)(\Delta \otimes \mathrm{id} \otimes \mathrm{id})(\varphi)$ and $(e \otimes \varphi)(\mathrm{id} \otimes \Delta \otimes \mathrm{id})(\varphi \otimes e)$ on physical states in the fourfold tensor product and comparing the coefficients, we may formulate $(5.5)$ as a relation for the $F_{P Q}[.$.$] which takes the form of a$ "pentagon identity [34]",

$$
F_{Q Q^{\prime}}\left[\begin{array}{cc}
K & Q^{\prime} \\
L & M
\end{array}\right] F_{P P^{\prime}}\left[\begin{array}{cc}
J & I \\
Q & M
\end{array}\right]=\sum_{R} F_{Q R}\left[\begin{array}{cc}
K & J \\
L & P
\end{array}\right] F_{P Q^{\prime}}\left[\begin{array}{cc}
R & I \\
L & M
\end{array}\right] F_{R P^{\prime}}\left[\begin{array}{cc}
J & I \\
K & Q^{\prime}
\end{array}\right] .
$$

Using the same procedure for the evaluation of $\varphi_{231}($ id $\otimes \Delta)(R)_{\varphi}$ and $R_{13} \varphi_{213} R_{12}$ relation (5.25) requires the "hexagon identity [34]"

$$
\begin{aligned}
& \sum_{P^{\prime}}(-1)^{P^{\prime}} q^{-\frac{1}{2} c_{P \prime}} F_{P^{\prime} Q}\left[\begin{array}{ll}
I & K \\
J & L
\end{array}\right] F_{P P^{\prime}}\left[\begin{array}{ll}
J & I \\
K & L
\end{array}\right] \\
& \quad=(-1)^{I+J+K+I-Q-P} q^{\frac{1}{2} c_{Q}+\frac{1}{2} c_{P}-\frac{1}{2} c_{I}-\frac{1}{2} c_{J}-\frac{1}{2} c_{K}-\frac{1}{2} c_{I} F_{P Q}}\left[\begin{array}{ll}
K & I \\
J & L
\end{array}\right]
\end{aligned}
$$

Here $c_{J}=(J+1) J$ and we used the relation

$$
\sum_{i^{\prime} j^{\prime}}\left(R^{I J}\right)_{i^{\prime} j^{\prime}, i j} C\left[\begin{array}{ccc}
J & I & K \\
j^{\prime} & i^{\prime} & k
\end{array}\right]_{q}=(-1)^{I+J-K} q^{\frac{1}{2} c_{K}-\frac{1}{2} c_{l}-\frac{1}{2} c_{j}} C\left[\begin{array}{ccc}
I & J & K \\
i & j & k
\end{array}\right]_{q} .
$$

A numerical solution of the restrictions (10.31) and (10.32) is explicitly known to be given by the fusion matrix,

$$
F_{P Q}\left[\begin{array}{ll}
J & I \\
K & L
\end{array}\right]=\left\{\begin{array}{ccc}
K & J & P \\
I & L & Q
\end{array}\right\}_{q}
$$

Here $\{::\}_{q}$ denote the $6 j$ symbols of $\mathrm{U}_{q}\left(\mathrm{sl}_{2}\right)$. In the case where $q$ is a root of unity it is well known that the corresponding identities for $6 j$ symbols (namely the $q$-analogs of Biedenharn-Elliot and Racah equations) restrict to summation over physical indices. The last identity (5.26) is also satisfied by this choice of $\varphi$.

The definition of $\varphi$ reduces to the identity when $\Delta(e)=e \otimes e$ and there is no truncation in the sum over representations. This situation prevails when $q$ is not a root of unity. In our situation $\varphi$ vanishes on a complement of the subspace $\hat{\mathrm{V}}_{i}^{I} \otimes\left(\hat{\mathrm{V}}_{j}^{J} \otimes \hat{\mathrm{V}}_{k}^{K}\right)$, and it maps this subspace onto $\left(\hat{\mathrm{V}}_{i}^{I} \otimes \hat{\mathrm{V}}_{j}^{J}\right) \otimes \hat{\mathrm{V}}_{k}^{K}$. This map is onto because it has an inverse $\varphi^{-1}$ which is given by the inverse fusion matrices. This inverse extends to a quasi-inverse on all of $\hat{V}_{i}^{I} \otimes \hat{V}_{j}^{\prime} \otimes \hat{V}_{k}^{K}$ by putting $\varphi^{-1}=$ (id $\otimes \Delta) \Delta(e) \varphi^{-1}$. By construction, this quasi-inverse has the defining properties 
(6.5) and (6.6) of a quasi-inverse. The property (6.9) is also true. This completes the proof of the existence of $\varphi$ with the properties that are required in a weak quasi-triangular quasi Hopf algebra and finishes the proof of the theorem.

It may be of interest to note that the fusion matrix $F$ which enters into the definition of $\varphi$ may be computed from the algebra of observables and its morphisms in the algebraic field theory approach $[26,33]$.

Example. The conformal Ising model, with multiplicities 1, 2, 3 assigned to the sectors with Virasoro lowest weight $0, \frac{1}{16}, \frac{1}{2}$, has the weak quasi-triangular quasi Hopf *-algebra $\mathscr{G}^{*}$ as a symmetry (in the sense of definition 3.2 ) which is canonically associated with $\mathrm{U}_{q}\left(\mathrm{sl}_{2}\right), q=i$, and its fields satisfy $\mathscr{G}^{*}$-covariant local braid relations.

The proof of this statement will be published in ref. [6].

\section{Supersymmetry}

It is instructive to consider supersymmetry as a special case of a quantum symmetry as described in this paper. Supersymmetry is not a purely internal symmetry, since it includes the space-time translation generators $P_{\mu}$ among its generators. This can be accommodated.

We adjoin fermionic parity $(-)^{F}$ to the generators of the supersymmetry algebra. It becomes a quasi-triangular Hopf algebra ("quantum group algebra") by defining co-product, co-unit and antipode as described below, and an $R$-element which reproduces the standard commutation/anticommutation relations. The coproduct is coassociative, therefore $\varphi=e \otimes e \otimes e$ and $\Delta(e)=e \otimes e$.

Apart from generators of groups, the supersymmetry algebra contains fermionic generators $Q_{\alpha}^{A}$ which anticommute with $(-)^{F}$. These fermionic generators obey anticommutation relations with Fermi field operators and commutation relations with Bose fields. Both are reproduced by the co-product defined below.

$$
\begin{aligned}
\Delta\left(Q_{\alpha}^{A}\right) & =Q_{\alpha}^{A} \otimes e+(-)^{F} \otimes Q_{\alpha}^{A} \\
R & =\frac{1}{2}\left[e \otimes e+(-)^{R} \otimes e+e \otimes(-)^{R}-(-)^{F} \otimes(-)^{R}\right] \\
\mathscr{S}\left(Q_{\alpha}^{A}\right) & =(-)^{F} Q_{\alpha}^{A} .
\end{aligned}
$$

Obviously, the co-product is not co-commutative. It may be called "graded co-commutative". The co-unit is the obvious one, $\epsilon\left(Q_{\alpha}^{A}\right)=0$, etc. All of Drinfeld's relations are satisfied. 
We are grateful to K.H. Rehren for several discussions. One of us (G.M.) would like to thank L. Faddeev and V. Pasquier for explaining their work. We were helped in our understanding of the co-module picture by a lecture of M. Karowski's in Hamburg [35].

V.S. wishes to thank R. Longo for the kind hospitality extended to him at the University of Rome.

\section{Appendix A}

\section{ADJOINT TRANSFORMATION LAW}

We start from the transformation law (3.15) of field operators under the algebra $\mathscr{G}^{*}, \mathrm{viz}$.

$$
U(\phi) \Psi_{i}^{I}=\sum_{p} \Psi_{m}^{I} \tau_{m i}^{l}\left(\phi_{p}^{1}\right) U\left(\phi_{p}^{2}\right)
$$

if $\Delta(\phi)=\Sigma_{p} \phi_{p}^{1} \otimes \phi_{p}^{2}$.

If we take the adjoint of this relation, the order of $U(\cdot)$ and $\Psi$ gets interchanged, so that the resulting relation tells us how to move representation operators $U(\cdot)$ through adjoints of field operators from right to left, rather than from left and right. For the special case of the quantum groups $\mathrm{U}_{q}\left(\mathrm{sl}_{2}\right)$ with $|q|=1$, the inverse relation was found in ref. [5],

$$
U(\phi) \Psi_{i}^{I *}=\sum_{p} \Psi_{m}^{l *} \tilde{\tau}_{m i}^{l}\left(\phi_{p}^{2}\right) U\left(\phi_{p}^{1}\right)
$$

where $\tilde{\tau}$ is the contragredient representation to $\tau$, defined with the help of the antipodal map $\mathscr{S}$,

$$
\tilde{\tau}(\phi)={ }^{\mathrm{t}} \tau\left(\mathscr{S}^{-1}(\phi)\right)
$$

We postulate general validity of the transformation law (A.2) for adjoints of field operators and determine what properties of the antipodal map $\mathscr{F}$ are required to assure this. It is immediately clear that $\mathscr{P}$ must be an antihomomorphism of $\mathscr{G}^{*}$ because otherwise $\tilde{\tau}$ does not have the representation property.

We assume that $\Delta$ is a $*$-homomorphism of algebras with the choice $(3.5)$ of *-operation on $\mathscr{G}^{*} \otimes \mathscr{G}^{*}$, so that

$$
\Delta\left(\phi^{*}\right)=\sum_{p} \phi_{p}^{2 *} \otimes \phi_{p}^{1 *}
$$


Substituting $\phi^{*}$ for $\phi$ in eq. (A.2) and taking its adjoint we find with the help of eq. (A.4) that transformation law (A.2) is equivalent to

$$
\Psi_{i}^{\prime} U(\phi)=\sum_{p} U\left(\phi_{p}^{2}\right) \Psi_{k}^{I} \tau_{k i}^{\prime}\left(\mathscr{S}^{-1}\left(\phi_{p}^{1}\right)\right)
$$

This relation tells us how one moves representation operators $U(\cdot)$ through field operators from right to left. Clearly, consistency of our postulate requires that one should obtain an identity if eq. (A.5) is substituted into eq. (A.1), or vice versa.

Let $\sum \Delta\left(\phi_{p}^{2}\right)=\sum_{q} \phi_{p q}^{21} \otimes \phi_{p q}^{2}$. Substituting (A.1) into eq. (A.5) yields an identity if

$$
\sum_{p g} \phi_{p q}^{21} \mathscr{P}^{-1}\left(\phi_{p}^{1}\right) \otimes \phi_{p q}^{22}=e \otimes \phi
$$

where $e$ is the unit element of $\mathscr{G}^{*}$, so that $\tau_{k i}^{I}(e)=\delta_{k i}$. Written more abstractly, this requirement reads

$$
m_{r}\left(\left(\mathscr{S}^{-1} \otimes \mathrm{id} \otimes \mathrm{id}\right)(\mathrm{id} \otimes \Delta) \Delta(\phi)\right)=e \otimes \phi
$$

with multiplication operator defined by

$$
m_{r}(\xi \otimes \eta \otimes \zeta)=\eta \xi \otimes \zeta
$$

Similarly, substitution of eq. (A.5) into eq. (A.1) yields an identity if

$$
m_{r}^{\prime}\left(\left(\mathrm{id} \otimes \mathscr{S}^{-1} \otimes \mathrm{id}\right)(\mathrm{id} \otimes \Delta) \Delta(\phi)\right)=\phi \otimes e,
$$

with multiplication operator defined by

$$
m_{r}^{\prime}(\xi \otimes \eta \otimes \zeta)=\zeta \otimes \eta \xi
$$

It is instructive to verify validity of these relations in the case when $\mathscr{G}^{*}$ is a group algebra. For group elements $a$ we have $\mathscr{P}(a)=a^{-1}$ and $\Delta(a)=a \otimes a$. Therefore

$$
(\text { id } \otimes \Delta) \Delta(a)=(\text { id } \otimes \Delta) a \otimes a=a \otimes a \otimes a,
$$

and

$$
\begin{aligned}
& m_{r}\left(\left(\mathscr{S}^{-1} \otimes \mathrm{id} \otimes \mathrm{id}\right) a \otimes a \otimes a\right)=m_{r}\left(a^{-1} \otimes a \otimes a\right)=a a^{-1} \otimes a=e \otimes a, \\
& m_{r}^{\prime}\left(\left(\mathrm{id} \otimes \mathscr{S}^{-1} \otimes \mathrm{id}\right) a \otimes a \otimes a\right)=m_{r}^{\prime}\left(a \otimes a^{-1} \otimes a\right)=a \otimes a^{-1} a=a \otimes e .
\end{aligned}
$$

This illustrates that the required properties of the antipodal map $\mathscr{S}$ are natural generalizations of the property that it maps a group element into its inverse. 


\section{Appendix B}

\section{CONSISTENCY WITH A NUMERICAL R-MATRIX}

We wish to examine what requirements are imposed by consistency of a quantum symmetry with local braid relations (4.3) with a numerical $R$-matrix. Both sides of eq. (4.3) will have to transform in the same way.

We multiply both sides of eq. (4.3) with $U(\phi)$ from the left, push $U(\cdot)$ through the fields using covariance property (3.15), and re-insert braid relations (4.3) on the left-hand side. Write

$$
\Delta(\phi)=\sum_{p} \phi_{p}^{1} \otimes \phi_{p}^{2}, \quad \Delta\left(\phi_{p}^{2}\right)=\sum_{q} \phi_{p q}^{21} \otimes \phi_{p q}^{22} .
$$

Then we find that we should have

$$
\sum_{p, q} \tau_{t m}^{J}\left(\phi_{p}^{1}\right) \tau_{u n}^{I}\left(\phi_{p q}^{21}\right) U\left(\phi_{p q}^{22}\right) R_{n m, i j}=\sum_{p, q} \tau_{u i}^{I}\left(\phi_{p}^{1}\right) \tau_{l j}^{J}\left(\phi_{p q}^{21}\right) U\left(\phi_{p q}^{22}\right) R_{u t, k l}
$$

We dropped superscripts $I J>$ on $\mathscr{R}$. Suppose that there exists an element $R$ in the algebra,

$$
R=\sum_{a} r_{a}^{1} \otimes r_{a}^{2} \in \mathscr{G}^{*} \otimes \mathscr{G}^{*}
$$

such that

$$
\mathscr{R}_{t u, s r}^{I J>}=\left(\tau^{\prime} \otimes \tau^{J}\right)_{u, s r}(R)=\sum_{a} \tau_{t s}^{I}\left(r_{a}^{1}\right) \tau_{u r}^{J}\left(r_{a}^{2}\right)
$$

Then requirement (B.1) will be satisfied if

$$
\sum_{p, q} r_{a}^{1} \phi_{p}^{1} \otimes r_{a}^{2} \phi_{p q}^{21} \otimes \phi_{p q}^{22}=\sum_{p g} \phi_{p q}^{21} r_{a}^{1} \otimes \phi_{p}^{1} r_{a}^{2} \otimes \phi_{p q}^{22}
$$

Written in more abstract form, this requirement reads

$$
(R \otimes e) \cdot(\mathrm{id} \otimes \Delta) \Delta(\phi)=\left(P_{12}(\mathrm{id} \otimes \Delta) \Delta(\phi)\right) \cdot(R \otimes e),
$$

where $P_{12}\left(a_{1} \otimes a_{2} \otimes a_{3}\right)=a_{2} \otimes a_{1} \otimes a_{3}$ and $\cdot$ is the product in $\mathscr{G}^{*} \otimes \mathscr{G}^{*} \otimes \mathscr{G}^{*}$.

\section{References}

[1] V.G. Drinfel'd, Quasi Hopf algebras and Knizhnik-Zamolodchikov equations, in Problems of modern quantum field theory, Proc. Alushta 1989, Research Reports in Physics (Springer, Heidelberg, 1989) 
[2] J. Fröhlich, Statistics of fields, the Yang-Baxter equation and the theory of knots and links, in Nonperturbative quantum field theory, ed, G.'t Hooft et al. (Plenum, New York, 1988)

[3] R. Dijkgraaf, V. Pasquier and P. Roche, Quasi-quantum groups related to orbifold models, in Proc. Int. Colloquium on Modern quantum field theory, TATA Institute of Fundamental Research, Bombay, January 1990

[4] D. Buchholz, G. Mack and I.T. Todorov, as reported in I. Todorov, in Proc. Conf. on Quantum Groups, Clausthal Zellerfeld, Germany, July 1989

[5] G. Mack and V. Schomerus, Commun. Math. Phys. 134 (1990) 139

[6] G. Mack and V. Schomerus, Phys. Lett. B267 (1991) 207

[7] V.G. Dringfel'd, Quantum groups, Proc. ICM (1987) 798

[8] S.L. Woronowicz, Commun. Math. Phys. (1987) 615; Twisted SU(2) group, An example of a noncommutative differential calculus, Publ. RIMSD-Kyoto 23 (1987) 117

[9] L.D. Faddeev, From integrable models to quantum groups, Schladming lectures 1990

[10] Yu.I. Manin, Quantum groups and non-commutative geometry, Proc. Int. Congr. Math., Berkeley $1(1986) 798$

[11] J. Wess and B. Zumino, Covariant differential calculus on the quantum hyperplane preprint CERN-TH 5697/90 (April 1990)

[12] L.D. Faddeev, N.Yu. Reshetikhin and L.A. Takhtajan, quantization of Lie groups and Lie algebras, preprint LOMI-E-14-87 (1987)

[13] L.A. Takhtajan, Adv. Stud Pure Math. 19 (1989)

[14] G. Moore and N.Yu. Reshetikhin, Nucl. Phys. B328 1989557

[15] N.Yu Reshetikhin and F. Smirnov, Commun. Math. Phys. 131 (1990) 157

[16] V. Pasquier and H. Saleur, Nucl. Phys. B330 (1990) 523

[17] H. Saleur and J.B. Zuber, Integrable lattice models and quantum groups, lectures at 1990 Spring School on String theory and quantum gravity, ICTP Trieste, April 1990

[18] H. Saleur, Phys. Rep. 184 (1989) 177

[19] L. Alvarez-Gaumé, C. Gomez and G. Sierra, Nucl. Phys. B319 (1989) 155; Phys. Lett. B220 (1989) 142; Nucl. Phys. B330 (1990) 347

[20] P. Furlan, A.Ch. Ganchev and V. Petkova, Quantum groups and fusion rules multiplicities, preprint INFN/AE-89/15 (1989)

[21] K. Gawedzki, Classical origin of quantum group symmetries in Wess-Zumino-Witten conformal field theories, IHES/P/90/92 (November 1990)

[22] G. Felder and $\mathrm{Ch}$. Wieczerkowski, Topological representations of the quantum group $\mathrm{U}_{q}\left(\mathrm{sl}_{2}\right)$, ETH-TH 90-52 (1990)

[23] N.Yu. Reshetikhin, L.A. Takhtajan and L.D. Faddeev, Algebra and Analysis 1 (1989) 178

[24] A.N. Kirillov and N. Reshetikbin, Representations of the algebra $\mathrm{U}_{a}(\mathrm{sl}(2))$, $q$-orthogonal polynomials and invariants of links, preprint LOMI E-9-88 (1988)

[25] G.G. Wick, E.P. Wigner and A.S. Wightman, Phys. Rev. 88 (1952) 101

[26] K. Fredenhagen, K.H. Rehren and B. Schroer, Commun. Math. Phys. 125 (1989) 201

[27] J. Fröhlich, F. Gabbiani and P.A. Marchetti, Braid statistics in three-dimensional local quantum theory, preprint ETH-TH/89-36 (1989); Superselection structure and statistics in three-dimensional local quantum theory preprint ETH-TH/89-22 (1989)

[28] D. Kastler, ed., The algebraic theory of superselection sectors, Introduction and recent results (World Scientific, Singapore, 1990)

[29] B. Schroer, private communication; K.H. Rehren, Field operators for anyons and plektons, preprint DESY 91-043 (May 1991)

[30] L. Hadjiivanov, R. Paunov and I.T. Todorov, Nucl. Phys. B356 (1991) 387

[31] A. Schirrmacher, J. Wess and B. Zumino, The two-parameter deformation of GL(2), its differential calculus and Lie algebra, Z. Phys. $C$, to be published

[32] G. Keller, Fusion rules of $U_{a}\left(\operatorname{sl}(2, \mathbb{C}), q^{m}=1\right.$, preprint MPI-PAE/PTh 47/90 (1990)

[33] G. Mack and V. Schomerus, Endomorphisms and quantum symmetry of the conformal Ising model, in The algebraic theory of superselection sectors, Introduction and recent results; ed. D. Kastler (World Scientific, Singapore, 1990)

[34] G. Moore and N. Seiberg, Phys. Lett. B212 (1988) 451; Commun. Math. Phys. 123 (1989) 77

[35] M. Karowski, Quantum groups and integrable models, lecture presented at DESY Hamburg, 1990

[36] I.M. Gelfand and G.E. Shilov, Generalized functions, Vol 1 (Academic Press, New York, 1964) 